\title{
Approximation of the thermally coupled MHD problem using a stabilized finite element method
}

\author{
Ramon Codina*, Noel Hernández \\ Universitat Politècnica de Catalunya, Jordi Girona 1-3, Edifici C1, 08034 Barcelona, Spain
}

\section{A R T I C L E I N F O}

\section{Article history:}

Received 23 September 2009

Received in revised form 14 September

2010

Accepted 1 November 2010

Available online 12 November 2010

\section{Keywords:}

Finite element methods

Stabilization

MHD

Thermal coupling

\begin{abstract}
A B S T R A C T
A numerical formulation to solve the MHD problem with thermal coupling is presented in full detail. The distinctive feature of the method is the design of the stabilization terms, which serve several purposes. First, convective dominated flows in the Navier-Stokes and the heat equation can be dealt with. Second, there is no restriction in the choice of the interpolation spaces of all the variables and, finally, flows highly coupled with the magnetic field can be accounted for. Different aspects related to the design of the final fully discrete and linearized algorithm are also discussed.
\end{abstract}

(c) 2010 Elsevier Inc. All rights reserved.

\section{Introduction}

The objective of this work is to present a finite element method for the approximation of the thermally-coupled magnetohydrodynamic (MHD) problem. We discuss several issues related to the time discretization, the linearization and the iterative coupling of the unknowns. However, our main concern is the design of a stabilization technique that allows one to use any continuous interpolation for all the unknowns, in particular, equal interpolation is allowed.

Considering first the thermally uncoupled case, in principle the unknowns involved are the magnetic field, the fluid velocity and the hydrodynamic pressure. However, to enforce the divergence free condition for the numerical approximation of the magnetic field we introduce a magnetic pseudo-pressure (whose exact value should be zero). This zero divergence condition is automatically satisfied at the continuous level for the transient problem if the initial magnetic field is solenoidal, but it is convenient to explicitly enforce it in the numerical approximation, especially for stationary problems. With the introduction of the magnetic pseudo-pressure we are left with a system of four equations with four unknowns. The 'augmented' approach used in this work is discussed for example in [19] in the context of MHD and more recently in [5] for the Maxwell equations. The same approach is used in $[14,29,24,23,28]$ (see also references therein). Other possibilities of enforcing the divergence free condition for the magnetic field are penalty strategies (see for example [1]) or the use of (weakly) divergence free interpolations based on Nédelec-type elements (described for example in [25,26]). These elements can also be used in combination with the augmented approach using a continuous approximation for the magnetic pseudo-pressure [28] so as to satisfy the adequate inf-sup condition between this scalar and the magnetic field. This condition also holds if an equal order discontinuous interpolation is used for both variables [17]. Nevertheless, there is also the possibility of relying on the mathematical structure of the equations and to expect that the original problem will already yield a magnetic field close

\footnotetext{
* Corresponding author.

E-mail addresses: ramon.codina@upc.edu (R. Codina), noelh@cimne.upc.edu (N. Hernández).
} 
enough to solenoidal. This is the idea followed in [22], which probably contains the first analysis of a finite element approximation to the MHD problem, and it is also used in [15], among other papers.

Having introduced the magnetic pseudo-pressure as a new unknown in the problem, its finite element approximation has several difficulties. First, there is the well known compatibility condition between the approximation spaces for the velocity and the pressure, but also for the approximation spaces for the magnetic field and the magnetic pseudo-pressure. Both conditions can be expressed in a standard inf-sup form [2]. There is also the problem of dealing with situations in which first order derivatives, both in the Navier-Stokes equations and in the equation for the magnetic field, dominate (from the numerical point of view) the second order terms that give an elliptic nature to the system of equations to be solved. These are the classical convection-dominated flow problems. Both the compatibility condition between interpolating spaces and the oscillations found in flows dominated by convection can be overcome by using stabilized finite element methods. First approaches in this direction can be found in [15] (without the introduction of the magnetic pseudo-pressure) and in [24,?] (where the magnetic pseudo-pressure is also introduced). However, another particular feature of the MHD problem are the couplings involved. In the magnetic problem, the coupling with the hydrodynamic problem comes from the convective term in the equation for the magnetic field, whereas in the Navier-Stokes equations the coupling with the magnetic problem comes from Lorentz's force. Our objective is to design a stabilized finite element method that takes these couplings into account.

The stabilized finite element method presented here is based on the two-scale decomposition of the unknowns into their finite element component and a subscale that cannot be captured by the finite element space. The format that we follow of this idea was introduced in [18]. In particular, the version for systems we employ here was already presented in [6]. A first version of our formulation, considering only the stationary and thermally uncoupled problem, can be found in [9].

The formulation is first designed for linear problems, and therefore our first concern is to devise a linearization technique for the fully coupled problem. For simplicity, we consider a fixed point method. Among the different possibilities, we identify the only one that leads to a linearized problem that is coercive, and thus guarantees existence and uniqueness of solution. This fixed point method is often used, but rarely justified. It is for this linearized problem that we propose a stabilized finite element method based on the subgrid scale concept. The important point is how to approximate the subgrid scales. We use the simplest approach of taking them proportional to a projection of the residual of the finite element approximation multiplied by the so called matrix of stabilization parameters. We consider two possibilities for the projection. The first is to take

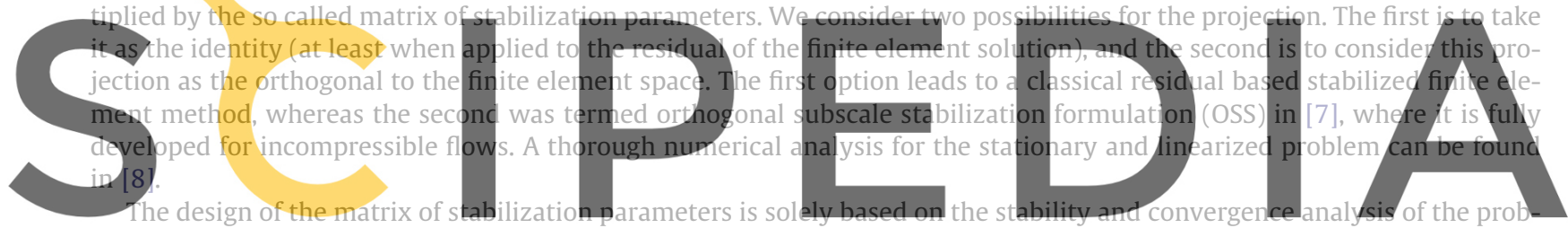

lem. This analysis will be presented in a situation as simple as possible, trying to avoid mathematical technicalities. It is not

tempt is made there to account neither for convection-dominated situations nor for the coupling effects) and in the design of the stabilization parameters. It also differs form the method proposed in [15] in the inclusion of the magnetic pseudo-pres sure and in the design of the stabilization parameters.

The paper is organized as follows. The problem to be solved is presented in the Section 2, including its strong and its variational forms. Issues not directly related to the finite element approximation are treated in Section 3, where a simple time integration scheme is described and linearization possibilities are discussed, starting with the identification of the only feasible fixed-point iteration for the thermally uncoupled MHD problem and then including the thermal coupling. The stabilization method is proposed and fully analyzed for the linearized stationary MHD problem in Section 4 . The scheme we finally propose is written in Section 5. Numerical examples are presented in Section 6 and conclusions are finally drawn in Section 7.

\section{Problem statement}

\subsection{Initial and boundary value problem}

Let $\Omega \subset \mathbb{R}^{d}(d=2$ or 3$)$ be a domain where we want to solve the thermally coupled MHD problem during the time interval $[0, T]$, the thermal coupling being modeled through Boussinesq's assumption. The unknowns of the problem are the fluid velocity $\boldsymbol{u}: \Omega \times(0, T) \rightarrow \mathbb{R}^{d}$, the pressure $p: \Omega \times(0, T) \rightarrow \mathbb{R}$, the magnetic induction (which we will simply call magnetic field) $\boldsymbol{B}: \Omega \times(0, T) \rightarrow \mathbb{R}^{d}$, the magnetic pseudo-pressure $r: \Omega \times(0, T) \rightarrow \mathbb{R}$ and the temperature $\vartheta: \Omega \times(0, T) \rightarrow \mathbb{R}$, which are solution of the system of partial differential equations:

$$
\partial_{t} \boldsymbol{u}+\boldsymbol{u} \cdot \nabla \boldsymbol{u}-v \Delta \boldsymbol{u}+\frac{1}{\rho} \nabla p-\frac{1}{\mu_{\mathrm{m}} \rho}(\nabla \times \boldsymbol{B}) \times \boldsymbol{B}+\mathbf{g} \beta \vartheta=\boldsymbol{f}_{\mathrm{f}}+\mathbf{g}\left[1+\beta \vartheta_{\mathrm{r}}\right],
$$

$\nabla \cdot \boldsymbol{u}=0$ 


$$
\begin{aligned}
& \partial_{\mathrm{t}} \boldsymbol{B}+\frac{1}{\mu_{\mathrm{m}} \sigma} \nabla \times(\nabla \times \boldsymbol{B})-\nabla \times(\boldsymbol{u} \times \boldsymbol{B})+\nabla r=\boldsymbol{f}_{\mathrm{m}}, \\
& \nabla \cdot \boldsymbol{B}=0, \\
& \partial_{\mathrm{t}} \vartheta+\boldsymbol{u} \cdot \nabla \vartheta-\frac{k_{\mathrm{t}}}{\rho c_{p}} \Delta \vartheta-\frac{1}{\rho c_{p} \mu_{\mathrm{m}}^{2} \sigma}|\nabla \times \boldsymbol{B}|^{2}-\frac{2 \mu_{\mathrm{f}}}{\rho c_{p}}\left|\nabla^{S} \boldsymbol{u}\right|^{2}=Q .
\end{aligned}
$$

In these equations, $\rho$ is the fluid density, $\mu_{\mathrm{f}}$ the fluid viscosity, $v=\mu_{\mathrm{f}} / \rho$ is the kinematic viscosity, $\mathrm{g}$ the gravity acceleration vector, $\beta$ the thermal expansion coefficient, $\boldsymbol{f}_{\mathrm{f}}$ the body force of the flow motion, $\vartheta_{\mathrm{r}}$ a known reference temperature, $\mu_{\mathrm{m}}$ the magnetic permeability, $\sigma$ the conductivity, $\boldsymbol{f}_{\mathrm{m}}$ a forcing term for the magnetic field (zero in the applications), $k_{\mathrm{t}}$ the thermal conduction coefficient, $c_{p}$ the specific heat at constant pressure, $\nabla^{S}$ is the symmetrical gradient operator and $Q$ the heating source. From now on, we consider that $\boldsymbol{f}_{\mathrm{f}}$ contains $\boldsymbol{g}\left[1+\beta \vartheta_{\mathrm{r}}\right]$. All physical properties will be considered constant.

In order to write the boundary conditions for problem (1)-(5), let us consider the disjoint splittings $\partial \Omega=\Gamma_{E, u} \cup \Gamma_{N, u}=$ $\Gamma_{E, B} \cup \Gamma_{N, B}=\Gamma_{E, \vartheta} \cup \Gamma_{N, \vartheta}$. Subscript $E$ refers to essential boundary conditions in the variational form to be presented, whereas $N$ refers to natural boundary conditions. The second subscript indicates the variable to which the condition is applied. If we denote with an overbar prescribed values, the boundary conditions to be considered for all time $t \in(0, T]$ are:

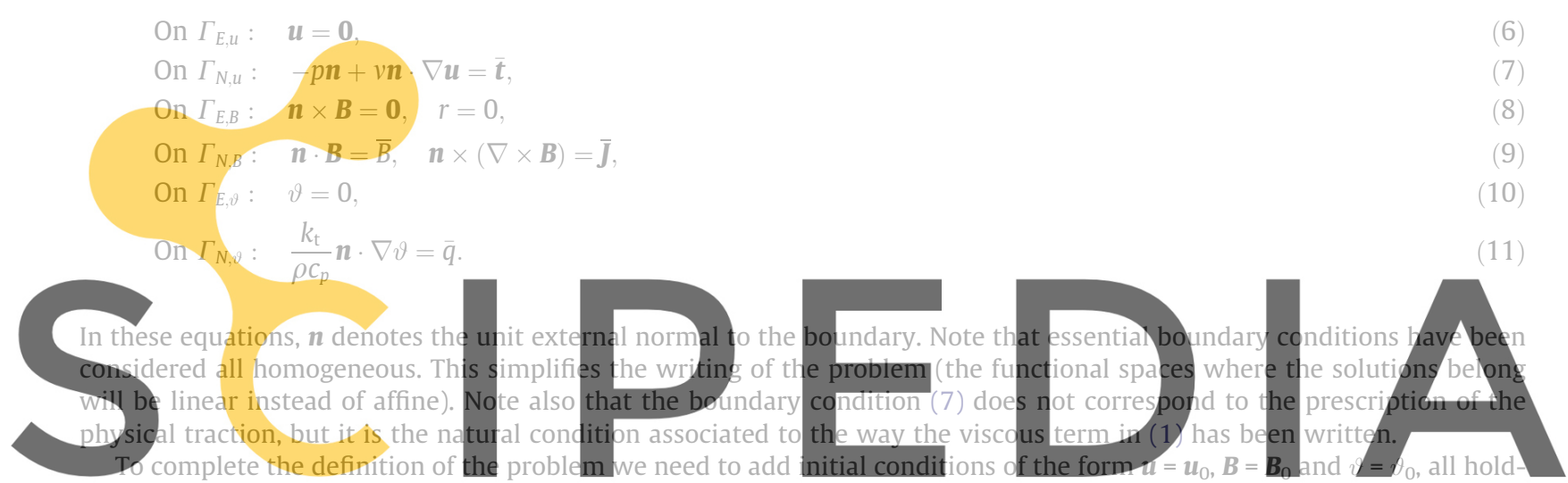

ing in the spatial domain $\Omega$ at $t=0$.

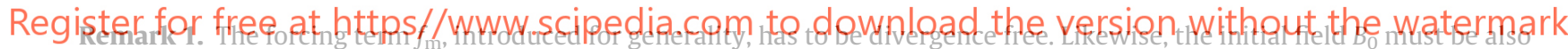

solenoidal. If one takes the divergence of (3) and uses the boundary conditions (8), it turns out that $r=0$. However, from the numerical point of view the introduction of $r$ will be very useful to enforce the zero divergence condition (4) while keeping the correct functional setting of the problem, as we will see in the next subsection.

Even though we will work with the dimensional form of the problem (1)-(5) to highlight the way it needs to be scaled, the equations can also be written in terms of the following dimensionless numbers:

$$
\begin{aligned}
& \operatorname{Re}:=\frac{\ell U}{v}, \quad \text { Reynolds number, } \\
& \operatorname{Pe}:=\frac{\ell U}{\kappa}, \quad \text { Péclet number, } \\
& \operatorname{Pr}:=\frac{v}{\kappa}, \quad \text { Prandtl number, } \\
& \mathrm{Gr}:=\frac{\beta|\boldsymbol{g}| \ell^{3} \delta \vartheta}{v^{2}}, \quad \text { Grashof number, } \\
& \operatorname{Re}_{\mathrm{m}}:=\mu_{\mathrm{m}} \sigma \ell U, \quad \text { Magnetic Reynolds number, } \\
& \mathrm{Ha}:=B \ell \sqrt{\frac{\sigma}{\rho v}}, \quad \text { Hartmann number, } \\
& \mathrm{S}:=\frac{B^{2}}{\mu_{\mathrm{m}} \rho U^{2}}, \quad \text { Coupling number, }
\end{aligned}
$$

where $\ell$ is a characteristic length of the problem, $U$ a characteristic velocity, $B$ a characteristic magnetic field and $\delta \vartheta$ a characteristic temperature difference, usually computed from temperature boundary values when these are not zero. These numbers are obviously not independent. For example, $\mathrm{Pe}=\operatorname{RePr}, \mathrm{Ha}=\left(\operatorname{Re}_{\mathrm{m}} \mathrm{S}\right)^{1 / 2}$. The data for the numerical examples presented in Section 6 will be given in terms of these numbers. 


\subsection{Weak form}

To write the weak form of (1)-(5) with the boundary conditions (6)-(11) let $\boldsymbol{v}, q, \boldsymbol{C}, s$ and $\psi$ be the test functions for $\boldsymbol{u}, p, \boldsymbol{B}$, $r$ and $\vartheta$, respectively. We consider them time-independent (time will be discretized using a finite difference scheme), $\boldsymbol{v}$ and $\psi$ are assumed the be zero on $\Gamma_{E, u}$ and $\Gamma_{E, \vartheta}$, respectively, and on $\Gamma_{E, B}$ it holds that $\boldsymbol{n} \times \boldsymbol{C}=\mathbf{0}$ and $s=0$.

Let us write $\langle f, g\rangle_{\omega}=\int_{\omega} f g$, where $f$ and $g$ are two generic functions defined on a region $\omega$ such that the integral of their product is well defined. No subscript will be used when $\omega=\Omega$. When $f, g \in L^{2}(\Omega)$, we will write $\langle f, g\rangle=(f, g)$. The norm in $L^{2}(\Omega)$ will be denoted by $\|f\|=(f, f)^{1 / 2}$. These symbols will be used for scalars, vectors or second order tensors.

Once Eqs. (1)-(5) are multiplied by the corresponding test functions, integrated over $\Omega$ and second order terms integrated by parts, the resulting variational form of the problem that we consider is

$$
\begin{aligned}
& \left(\partial_{t} \boldsymbol{u}, \boldsymbol{v}\right)+A_{u u}(\boldsymbol{u}, \boldsymbol{u}, \boldsymbol{v})+A_{u B}(\boldsymbol{B}, \boldsymbol{B}, \boldsymbol{v})+A_{u \vartheta}(\vartheta, \boldsymbol{v})-b_{u}(p, \boldsymbol{v})=L_{u}(\boldsymbol{v}), \\
& b_{u}(q, \boldsymbol{u})=0 \\
& \left(\partial_{t} \boldsymbol{B}, \boldsymbol{C}\right)+A_{B u}(\boldsymbol{u}, \boldsymbol{B}, \boldsymbol{C})+A_{B B}(\boldsymbol{B}, \boldsymbol{C})+b_{B}(r, \boldsymbol{C})=L_{B 1}(\boldsymbol{C}), \\
& b_{B}(s, \boldsymbol{B})=L_{B 2}(s),
\end{aligned}
$$

$$
\left(\partial_{t} \vartheta, \psi\right)+A_{\vartheta u, 1}(\boldsymbol{u}, \vartheta, \psi)+A_{\vartheta u, 2}(\boldsymbol{u}, \boldsymbol{u}, \psi)+A_{\vartheta}(\boldsymbol{B}, \boldsymbol{B}, \psi)+A_{\vartheta \vartheta}(\vartheta, \psi)=L_{\vartheta}(\psi),
$$

which must hold for all test functions $\boldsymbol{v}, q, \boldsymbol{C}, s$ and $\psi$ in the functional spaces indicated next.

The different multilinear forms appearing in (12)-(16) are given by

$$
\begin{aligned}
& A_{u u}\left(\boldsymbol{u}_{1}, \boldsymbol{u}_{2}, \boldsymbol{v}\right)=\left\langle\boldsymbol{v}, \boldsymbol{u}_{1} \cdot \nabla \boldsymbol{u}_{2}\right\rangle+v\left(\nabla \boldsymbol{v}, \nabla \boldsymbol{u}_{2}\right), \\
& A_{u B}\left(\boldsymbol{B}_{1}, \boldsymbol{B}_{2}, \boldsymbol{v}\right)=-\frac{1}{\mu_{\mathrm{m}} \rho}\left\langle\boldsymbol{v},\left(\nabla \times \boldsymbol{B}_{1}\right) \times \boldsymbol{B}_{2}\right\rangle,
\end{aligned}
$$
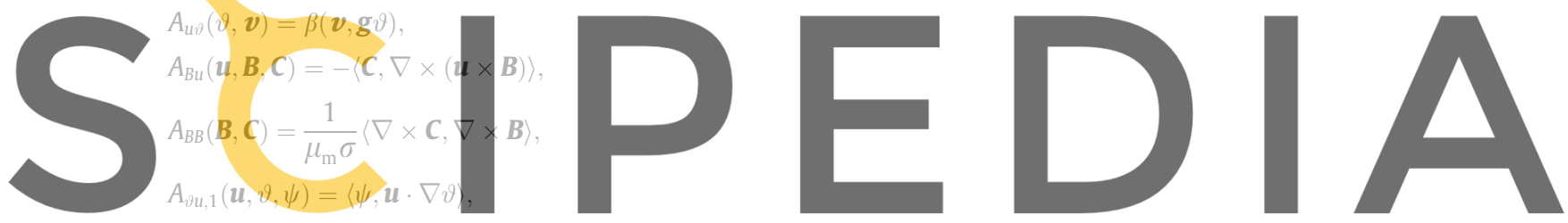

$A_{\vartheta u, 2}\left(\boldsymbol{u}_{1}, \boldsymbol{u}_{2}, \psi\right)=-\frac{2 \mu_{\mathrm{f}}}{2}\left\langle\psi, \nabla^{S} \boldsymbol{u}_{1}: \nabla^{S} \boldsymbol{u}_{2}\right.$

Register for free at http\$p/wWw.scipedia.com to download the version without the watermark

$$
\begin{aligned}
& A_{\vartheta B}\left(\boldsymbol{B}_{1}, \boldsymbol{B}_{2}, \psi\right)=-\frac{1}{\rho c_{p} \mu_{\mathrm{m}}^{2} \sigma}\left\langle\psi,\left(\nabla \times \boldsymbol{B}_{1}\right) \cdot\left(\nabla \times \mathbb{B}_{2}\right)\right\rangle, \\
& A_{\vartheta \vartheta}(\vartheta, \psi)=\frac{k_{\mathrm{t}}}{\rho c_{p}}(\nabla \psi, \nabla \vartheta), \\
& b_{u}(q, \boldsymbol{v})=\frac{1}{\rho}(q, \nabla \cdot \boldsymbol{v}), \\
& b_{B}(s, \boldsymbol{C})=(\nabla s, \boldsymbol{C}), \\
& L_{u}(\boldsymbol{v})=\left\langle\boldsymbol{v}, \boldsymbol{f}_{\mathrm{f}}\right\rangle+\langle\boldsymbol{v}, \overline{\boldsymbol{t}}\rangle_{\Gamma_{N, u}}, \\
& L_{B 1}(\boldsymbol{C})=\left(\boldsymbol{C}, \boldsymbol{f}_{\mathrm{m}}\right)+\langle\boldsymbol{v}, \overline{\boldsymbol{J}}\rangle_{\Gamma_{N, B}}, \\
& L_{B 2}(s)=\langle s, \bar{B}\rangle_{\Gamma_{N, B}}, \\
& L_{\vartheta}(\psi)=\langle\psi, Q\rangle+\langle\psi, \bar{q}\rangle_{\Gamma_{N, T}} .
\end{aligned}
$$

If we consider the functional spaces

$$
\begin{aligned}
& V_{u}=\left\{\boldsymbol{v} \in H^{1}(\Omega)^{d} \mid \boldsymbol{v}=\mathbf{0} \text { on } \Gamma_{E, u}\right\}, \\
& V_{p}=\left\{q \in L^{2}(\Omega) \mid \int_{\Omega} q=0 \text { if } \Gamma_{N, u}=\emptyset\right\}, \\
& V_{B}=\left\{\boldsymbol{C} \in H(\operatorname{curl}, \Omega) \mid \boldsymbol{n} \times \boldsymbol{C}=\mathbf{0} \text { on } \Gamma_{E, B}\right\}, \\
& V_{r}=\left\{s \in H^{1}(\Omega) \mid s=0 \text { on } \Gamma_{E, B}\right\}, \\
& V_{\vartheta}=\left\{\psi \in H^{1}(\Omega) \mid \psi=0 \text { on } \Gamma_{E, \vartheta}\right\},
\end{aligned}
$$


all the multilinear forms introduced, except $A_{\vartheta u, 2}\left(\boldsymbol{u}_{1}, \boldsymbol{u}_{2}, \psi\right)$ and $A_{\vartheta B}\left(\boldsymbol{B}_{1}, \boldsymbol{B}_{2}, \psi\right)$, are well defined and continuous for

$$
\begin{aligned}
& \boldsymbol{u}_{1}, \boldsymbol{u}_{2}, \boldsymbol{u} \in L^{2}\left(0, T ; V_{u}\right), \quad \boldsymbol{v} \in V_{u}, \\
& p \in \mathcal{D}^{\prime}\left(0, T ; V_{p}\right), \quad q \in V_{p}, \\
& \boldsymbol{B}_{1}, \boldsymbol{B}_{2}, \boldsymbol{B} \in L^{2}\left(0, T ; V_{B}\right), \quad \boldsymbol{C} \in V_{B}, \\
& r \in \mathcal{D}^{\prime}\left(0, T ; V_{r}\right), \quad s \in V_{r}, \\
& \vartheta \in L^{2}\left(0, T ; V_{\vartheta}\right), \quad \psi \in V_{\vartheta} .
\end{aligned}
$$

In these expressions, $L^{2}(0, T ; X)$ denotes the set of mappings defined on $\Omega \times(0, T)$ such that their $X$-spatial norm is an $L^{2}(0, T)$ function. Similarly, $\mathcal{D}^{\prime}(0, T ; X)$ denotes the set of mappings for which their $X$-spatial norm is a distribution in time.

If $A_{\vartheta u, 2}\left(\boldsymbol{u}_{1}, \boldsymbol{u}_{2}, \psi\right)$ and $A_{\vartheta B}\left(\boldsymbol{B}_{1}, \boldsymbol{B}_{2}, \psi\right)$ need to be taken into account, one should require $\nabla^{S} \boldsymbol{v} \in W^{1,4}(\Omega)^{d \times d}$ in the definition of $V_{u}$ and $\nabla \times \boldsymbol{C} \in L^{4}(\Omega)^{d}$ in the definition of $V_{B}$. It is also assumed that $\boldsymbol{f}_{\mathrm{f}} \in V_{u}^{\prime}, \boldsymbol{f}_{\mathrm{m}} \in V_{B}^{\prime}$ and $Q \in V_{\vartheta}^{\prime}$ (a.e. in time), where $X^{\prime}$ denotes the topological dual of a function space $X$.

To close this section, let us note that (12)-(16) can be written as a single variational equation of the form

$$
M\left(\partial_{t} \boldsymbol{U}, \boldsymbol{V}\right)+A(\boldsymbol{U}, \boldsymbol{V})=L(\boldsymbol{V}),
$$

where

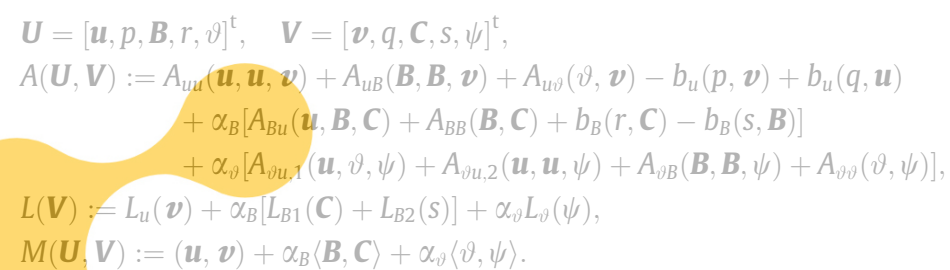

The scaling coefficients $\alpha_{B}$ and $\alpha_{\vartheta}$ need to be introduced to make the semilinear form $A(\boldsymbol{U}, \boldsymbol{V})$ and the linear form $L(\mathbb{V})$ dimen-
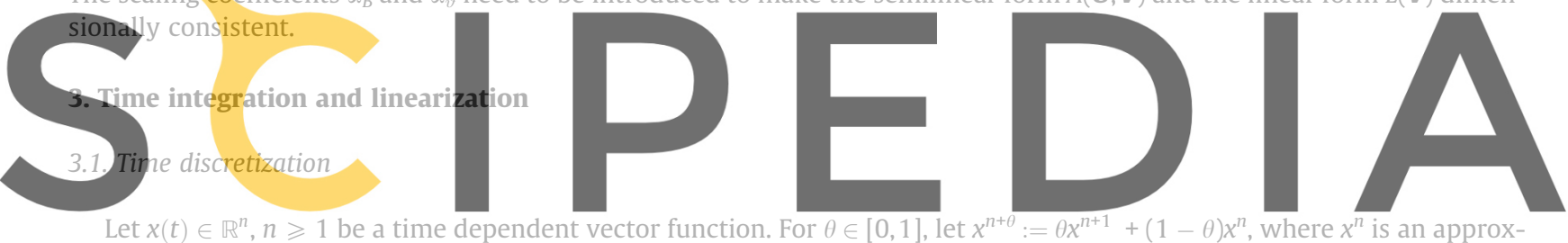

imation to $x\left(t^{n}\right)$ and $t^{n}:=n \delta t, \delta t$ being the time step size of a uniform partition of $[0, T]$.

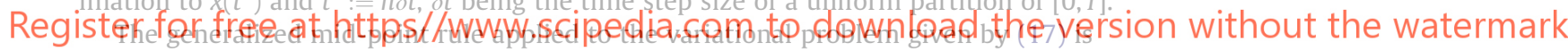

$$
M\left(\delta_{t} \boldsymbol{U}^{n}, \boldsymbol{V}\right)+A\left(\boldsymbol{U}^{n+\theta}, \boldsymbol{V}\right)=L(\boldsymbol{V}),
$$

where $\delta_{t} \boldsymbol{U}^{n}=\delta t^{-1}\left(\boldsymbol{U}^{n+1}-\boldsymbol{U}^{n}\right)=(\theta \delta t)^{-1}\left(\boldsymbol{U}^{n+\theta}-\boldsymbol{U}^{n}\right)$. This is the time discretization of the problem that we will consider, although other time integration schemes could be also applied to obtain the final fully discrete and linearized problem.

\subsection{Linearization and block-iterative coupling}

In this section we present a linearization for the time discrete problem (18). If fact, we are also interested in uncoupling the calculation of the temperature from the rest of unknowns.

\subsubsection{Linearization of the stationary MHD problem}

The first issue we consider is the linearization of the nonlinear terms in (18). To this end, it is enough to consider the thermally uncoupled and stationary problem. If we introduce now the vector of unknowns $\boldsymbol{U}=[\boldsymbol{u}, p, \boldsymbol{B}, r]^{\mathrm{t}}$ and the corresponding test functions $\boldsymbol{V}=[\boldsymbol{v}, q, \boldsymbol{C}, s]^{\mathrm{t}}$, the variational problem can be written as

$$
A(\boldsymbol{U}, \boldsymbol{V})=L(\boldsymbol{V}) .
$$

Taking the scaling coefficient as $\alpha_{B}=1 /\left(\mu_{\mathrm{m}} \rho\right)$ now we have

$$
\begin{aligned}
A(\boldsymbol{U}, \boldsymbol{V})= & \langle\boldsymbol{v}, \boldsymbol{u} \cdot \nabla \boldsymbol{u}\rangle+v(\nabla \boldsymbol{v}, \nabla \boldsymbol{u})-\frac{1}{\mu_{\mathrm{m}} \rho}\langle\boldsymbol{v},(\nabla \times \boldsymbol{B}) \times \boldsymbol{B}\rangle-\frac{1}{\rho}(p, \nabla \cdot \boldsymbol{v})+\frac{1}{\rho}(q, \nabla \cdot \boldsymbol{u}) \\
& +\frac{1}{\mu_{\mathrm{m}} \rho}\left[-\langle\boldsymbol{C}, \nabla \times(\boldsymbol{u} \times \boldsymbol{B})\rangle+\frac{1}{\mu_{\mathrm{m}} \sigma}(\nabla \times \boldsymbol{C}, \nabla \times \boldsymbol{B})+(\nabla r, \boldsymbol{C})-(\nabla s, \boldsymbol{B})\right] .
\end{aligned}
$$

The simplest way to linearize the problem is by a fixed point treatment of the quadratic terms. Let us assume we have an estimate for the velocity and the magnetic field at iteration $k, \boldsymbol{u}^{k}$ and $\boldsymbol{B}^{k}$, respectively, and we have to compute these fields 
at iteration $k+1$. If $e_{i}(k)=k$ or $e_{i}(k)=k+1$ and $e_{i}^{\prime}(k)=2 k+1-e_{i}(k)$, the approximation of $A(\boldsymbol{U}, \boldsymbol{V})$ at iteration $k+1$ using the fixed point method may be written as

$$
\begin{aligned}
A^{k+1}(\boldsymbol{U}, \boldsymbol{V})= & \left\langle\boldsymbol{v},\left(\boldsymbol{u}^{e_{1}(k)} \cdot \nabla\right) \boldsymbol{u}_{1}^{e_{1}^{\prime}(k)}\right\rangle+v\left(\nabla \boldsymbol{v}, \nabla \boldsymbol{u}^{k+1}\right)-\frac{1}{\mu_{\mathrm{m}} \rho}\left\langle\boldsymbol{v},\left(\nabla \times \boldsymbol{B}^{e_{2}(k)}\right) \times \boldsymbol{B}^{e_{2}^{\prime}(k)}\right\rangle-\frac{1}{\rho}\left\langle p^{k+1}, \nabla \cdot \boldsymbol{v}\right\rangle+\frac{1}{\rho}\left\langle q, \nabla \cdot \boldsymbol{u}^{k+1}\right\rangle \\
& +\frac{1}{\mu_{\mathrm{m}} \rho}\left[-\left\langle\boldsymbol{C}, \nabla \times\left(\boldsymbol{u}^{e_{3}(k)} \times \boldsymbol{B}^{e_{3}^{\prime}(k)}\right)\right\rangle+\frac{1}{\mu_{\mathrm{m}} \sigma}\left(\nabla \times \boldsymbol{C}, \nabla \times \boldsymbol{B}^{k+1}\right)+\left(\nabla r^{k+1}, \boldsymbol{C}\right)-\left(\nabla s, \boldsymbol{B}^{k+1}\right)\right] .
\end{aligned}
$$

In order to have a stable problem at each iteration, we should guarantee that $A^{k+1}\left(\boldsymbol{U}^{k+1}, \boldsymbol{U}^{k+1}\right) \geqslant 0$, which leads to conditions

$$
\begin{aligned}
& \int_{\Omega} \boldsymbol{u}^{k+1} \cdot\left(\boldsymbol{u}^{e_{1}(k)} \cdot \nabla\right) \boldsymbol{u}^{e_{1}^{\prime}(k)} \geqslant 0, \\
& \int_{\Omega}\left[-\boldsymbol{u}^{k+1} \cdot\left(\nabla \times \boldsymbol{B}^{e_{2}(k)}\right) \times \boldsymbol{B}^{e_{2}^{\prime}(k)}-\boldsymbol{B}^{k+1} \cdot \nabla \times\left(\boldsymbol{u}^{e_{3}(k)} \times \boldsymbol{B}^{e^{\prime}(k)}\right)\right] \geqslant 0 .
\end{aligned}
$$

When $\nabla \cdot \boldsymbol{u}^{k}=0, \nabla \cdot \boldsymbol{B}^{k}=0$, these conditions hold only if $e_{1}(k)=k$ (as it is well known), $e_{2}(k)=k+1$ and $e_{3}(k)=k+1$. Therefore, calling $\boldsymbol{a} \equiv \boldsymbol{u}^{k}, \boldsymbol{u} \equiv \boldsymbol{u}^{k+1}, \boldsymbol{b} \equiv \boldsymbol{B}^{k}, \boldsymbol{B} \equiv \boldsymbol{B}^{k+1}$, the only fixed point linearization of the problem that is stable is

$$
\begin{aligned}
& (\boldsymbol{a} \cdot \nabla) \boldsymbol{u}-v \Delta \boldsymbol{u}+\frac{1}{\rho} \nabla p-\frac{1}{\mu_{\mathrm{m}} \rho}(\nabla \times \boldsymbol{B}) \times \boldsymbol{b}=\boldsymbol{f}_{\mathrm{f}}, \\
& \nabla \cdot \boldsymbol{u}=0, \\
& \frac{1}{\mu_{\mathrm{m}} \sigma} \nabla \times(\nabla \times \boldsymbol{B})-\nabla \times(\boldsymbol{u} \times \boldsymbol{b})+\nabla r=\boldsymbol{f}_{\mathrm{m}}, \\
& \nabla \cdot \boldsymbol{B}=0 .
\end{aligned}
$$

This is the problem for which the stabilized finite element method will be presented in Section 4. Note that the problem needs to be solved in a coupled way, without the possibility to segregate the fluid mechapics problem from the magnetic one. The same would happen if instead of a simple fixed-point linearization scheme, the classical Newton-Raphson/inearization is used.

2.2. Full coupling and block-iterative coupling

us consider again the stationary problem, but now
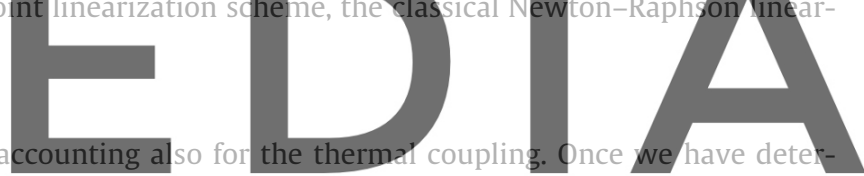

mined how to deal with the nonlinearity arising because of the velocity-magnetic field coupling, the thermal coupling is easy to treat, since the temperature term in the momentum equation is linear. We may consider either a full coupling or a block

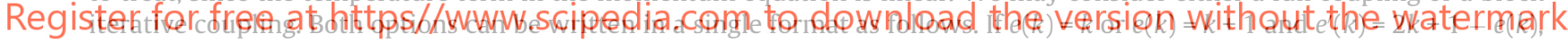
we introduce the fully linearized and coupled problem

$$
\begin{aligned}
& A_{u u}\left(\boldsymbol{u}^{k}, \boldsymbol{u}^{k+1}, \boldsymbol{v}\right)+A_{u B}\left(\boldsymbol{B}^{k+1}, \boldsymbol{B}^{k}, \boldsymbol{v}\right)+A_{u \vartheta}\left(\vartheta^{e(k)}, \boldsymbol{v}\right)-b_{u}\left(p^{k+1}, \boldsymbol{v}\right)=L_{u}(\boldsymbol{v}), \\
& b_{u}\left(q, \boldsymbol{u}^{k+1}\right)=0 \\
& A_{B u}\left(\boldsymbol{u}^{k+1}, \boldsymbol{B}^{k}, \boldsymbol{C}\right)+A_{B B}\left(\boldsymbol{B}^{k+1}, \boldsymbol{C}\right)+b_{B}\left(r^{k+1}, \boldsymbol{C}\right)=L_{B 1}(\boldsymbol{C}), \\
& b_{B}\left(s, \boldsymbol{B}^{k+1}\right)=L_{B 2}(S), \\
& A_{\vartheta u, 1}\left(\boldsymbol{u}^{e^{\prime}(k)}, \vartheta^{k+1}, \psi\right)+A_{\vartheta \vartheta}\left(\vartheta^{k+1}, \psi\right)=L_{\vartheta}^{k}(\psi),
\end{aligned}
$$

where

$$
L_{\vartheta}^{k}(\psi)=L_{\vartheta}(\psi)-A_{\vartheta u, 2}\left(\boldsymbol{u}^{e^{\prime}(k)}, \boldsymbol{u}^{e^{\prime}(k)}, \psi\right)-A_{\vartheta B}\left(\boldsymbol{B}^{e^{\prime}(k)}, \boldsymbol{B}^{e^{\prime}(k)}, \psi\right) .
$$

It is clear that when $e(k)=k+1$ (and thus $e^{\prime}(k)=k$ ), the problem needs to be solved for $\boldsymbol{u}^{k+1}, p^{n+1}, \boldsymbol{B}^{k+1}, s^{k+1}$ and $\vartheta^{k+1}$ in a coupled way. The production of heat given by $A_{\vartheta u, 2}\left(\boldsymbol{u}^{e^{\prime}(k)}, \boldsymbol{u}^{e^{\prime}(k)}, \psi\right)$ and $A_{\vartheta B}\left(\boldsymbol{B}^{e^{\prime}(k)}, \boldsymbol{B}^{e^{\prime}(k)}, \psi\right)$ needs to be evaluated at the previous iteration (unless a Newton-Raphson-type strategy is used). On the other hand, when $e(k)=k$ the problem can be solved first for $\boldsymbol{u}^{k+1}, p^{n+1}, \boldsymbol{B}^{k+1}$ and $r^{k+1}$. Once these variables are computed, temperature may be updated by solving (21). In this case, it is possible to use the variables $\boldsymbol{u}^{k+1}$ and $\boldsymbol{B}^{k+1}$ just computed, thus leading to a Gauss-Seidel-type coupling. Of course, a Jacobi coupling, in which $L_{\vartheta}^{k}$ in (22) is evaluated with $\boldsymbol{u}^{k}$ and $\boldsymbol{B}^{k}$, would also be possible. However, the computational effort is the same, and convergence is known to be faster for Gauss-Seidel-type schemes (see [4] for further discussion).

\subsection{Time discrete and linearized scheme}

The next step is to consider the time discrete problem using the mid-point rule together with the linearization scheme described previously. The combination is straightforward (see Section 5 below). 
For implementation purposes, it is very convenient to write the problem to be solved as a time-discrete system of linear convection-diffusion-reaction equations. Let us consider the case $e(k)=k+1$ in (20), the case $e(k)=k$ being similar, and let us call $\boldsymbol{a} \equiv \boldsymbol{u}^{n+\theta, k}, \boldsymbol{u} \equiv \boldsymbol{u}^{n+\theta, k+1}, \boldsymbol{b} \equiv \boldsymbol{B}^{n+\theta, k}, \boldsymbol{B} \equiv \boldsymbol{B}^{n+\theta, k+1}, \vartheta \equiv \vartheta^{n+\theta, k+1}$ and $\delta_{t} f^{n}=(\theta \delta t)^{-1}\left(f-f^{n}\right)$ for any of the unknowns $f$. Here and below the first superscript denotes the time step level and the second the iteration counter. The differential equations associated to the time discrete and linearized scheme can be written as

$$
\begin{aligned}
& \delta_{t} \boldsymbol{u}+(\boldsymbol{a} \cdot \nabla) \boldsymbol{u}-v \Delta \boldsymbol{u}+\frac{1}{\rho} \nabla p-\frac{1}{\mu_{\mathrm{m}} \rho}(\nabla \times \boldsymbol{B}) \times \boldsymbol{b}+\mathbf{g} \beta \vartheta=\boldsymbol{f}_{\mathrm{f}}, \\
& \nabla \cdot \boldsymbol{u}=0, \\
& \delta_{t} \boldsymbol{B}+\frac{1}{\mu_{\mathrm{m}} \sigma} \nabla \times(\nabla \times \boldsymbol{B})-\nabla \times(\boldsymbol{u} \times \boldsymbol{b})+\nabla r=\boldsymbol{f}_{\mathrm{m}}, \\
& \nabla \cdot \boldsymbol{B}=0, \\
& \delta_{t} \vartheta+\boldsymbol{a} \cdot \nabla \vartheta-\frac{k_{\mathrm{t}}}{\rho c_{p}} \Delta \vartheta=Q_{\mathrm{tot}},
\end{aligned}
$$

where

$$
Q_{\text {tot }}=Q-\frac{1}{\rho c_{p} \mu_{\mathrm{m}}^{2} \sigma}|\nabla \times \boldsymbol{b}|^{2}-\frac{2 \mu_{\mathrm{f}}}{\rho c_{p}}\left|\nabla^{S} \boldsymbol{a}\right|^{2} .
$$

The problem considered can be written as the vector differential equation

$$
\boldsymbol{M} \delta_{t} \boldsymbol{U}+\mathcal{L}(\boldsymbol{U})=\boldsymbol{F} \text { in } \Omega
$$

where $\boldsymbol{M}=\operatorname{diag}\left(\boldsymbol{I}, 0, \alpha_{B} \boldsymbol{I}, 0, \alpha_{\vartheta}\right)$, I being the $d \times d$ identity, $\delta_{t} \boldsymbol{U}=(\delta t)^{-1}\left(\boldsymbol{U}-\boldsymbol{U}^{m}\right)$, with $\boldsymbol{U}^{n}$ known, $\boldsymbol{F}=\left[\boldsymbol{f}_{\mathrm{f}}, 0, \alpha_{B} \boldsymbol{f}_{\mathrm{m}}, 0, \alpha_{\vartheta} Q_{\mathrm{tot}}\right]^{\mathrm{t}}$ is a known vector of $n_{\mathrm{unk}}=2 d+3$ components and the (scaled) operator $\mathcal{L}$ is given by

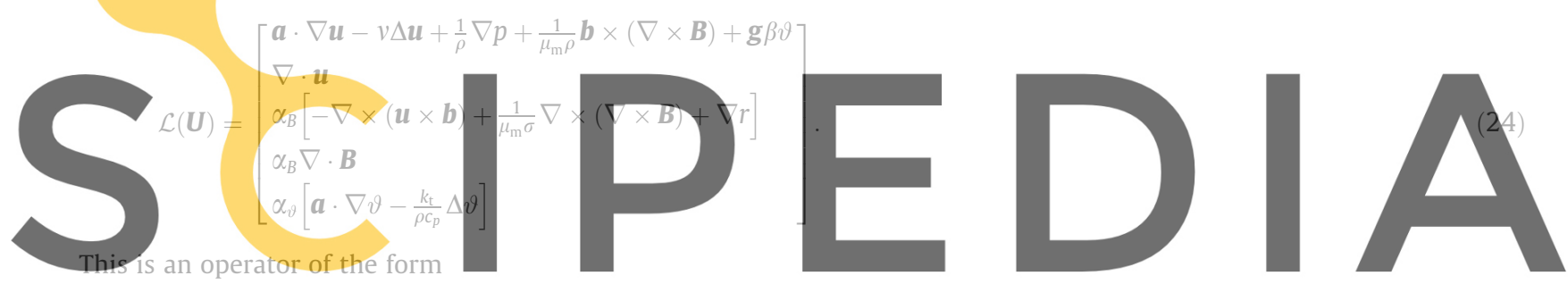

$\mathcal{L}(\boldsymbol{U}):=\boldsymbol{A}_{i} \partial_{i} \boldsymbol{U}-\partial_{i}\left(\boldsymbol{K}_{i j} \partial_{j} \boldsymbol{U}\right)$

Register for free at https//www scipedia.com to download theversion without the watermark

follows, repeated indexes imply summation up to the number of spatial dimensions and $\partial_{i}$ is the partial derivative with respect to the Cartesian coordinate $x_{i}$.

Let matrices $\boldsymbol{A}_{i}$ be split as $\boldsymbol{A}_{i}=\boldsymbol{A}_{i}^{\mathrm{c}}+\boldsymbol{A}_{i}^{\mathrm{f}}$, where $\boldsymbol{A}_{i}^{\mathrm{c}}$ is the part of the convection matrices which is not integrated by parts and $\boldsymbol{A}_{i}^{\mathrm{f}}$ the part that is integrated by parts. In our case, matrices $\boldsymbol{A}_{i}^{\mathrm{f}}$ come from the first order derivatives of the hydrodynamic pressure $p$. It would be also possible to integrate by parts the first order derivatives corresponding to the terms $\boldsymbol{u} \cdot \nabla \boldsymbol{u}$ and $\nabla \times(\boldsymbol{u} \times \boldsymbol{B})$.

The weak form of the problem supplied with the appropriate homogeneous boundary conditions can be written again as the time discrete and linearized counterpart of (17):

$$
M\left(\delta_{t} \boldsymbol{U}, \boldsymbol{V}\right)+A^{\operatorname{lin}}(\boldsymbol{U}, \boldsymbol{V})=L(\boldsymbol{V})
$$

where

$$
A^{\operatorname{lin}}(\boldsymbol{U}, \boldsymbol{V}):=\left(\boldsymbol{V}, \boldsymbol{A}_{i}^{\mathrm{c}} \partial_{i} \boldsymbol{U}\right)-\left(\left(\boldsymbol{A}_{i}^{\mathrm{f}}\right)^{\mathrm{t}} \partial_{i} \boldsymbol{V}, \boldsymbol{U}\right)+\left(\partial_{i} \boldsymbol{V}, \boldsymbol{K}_{i j} \partial_{j} \boldsymbol{U}\right)
$$

and $L(\boldsymbol{V}):=(\boldsymbol{V}, \boldsymbol{F})$ plus boundary contributions that depend on the problem. For the particular case of MHD, taking $\alpha_{B}=1 /$ $\left(\mu_{\mathrm{m}} \rho\right)$ :

$$
\begin{aligned}
A^{\operatorname{lin}}(\boldsymbol{U}, \boldsymbol{V})= & \langle\boldsymbol{v}, \boldsymbol{a} \cdot \nabla \boldsymbol{u}\rangle+v(\nabla \boldsymbol{v}, \nabla \boldsymbol{u})-\frac{1}{\mu_{\mathrm{m}} \rho}\langle\boldsymbol{v},(\nabla \times \boldsymbol{B}) \times \boldsymbol{b}\rangle-\frac{1}{\rho}(p, \nabla \cdot \boldsymbol{v})+\frac{1}{\rho}(q, \nabla \cdot \boldsymbol{u}) \\
& +\frac{1}{\mu_{\mathrm{m}} \rho}\left[-\langle\boldsymbol{C}, \nabla \times(\boldsymbol{u} \times \boldsymbol{b})\rangle+\frac{1}{\mu_{\mathrm{m}} \sigma}(\nabla \times \boldsymbol{C}, \nabla \times \boldsymbol{B})+\langle\nabla r, \boldsymbol{C}\rangle-\langle\nabla s, \boldsymbol{B}\rangle\right]
\end{aligned}
$$

From now on, we will redefine $p \leftarrow p / \rho$, that is to say, we will work with the kinematic pressure. 


\section{Stabilized formulation for the stationary, linearized and thermally uncoupled problem}

\subsection{Stability of the Galerkin approximation}

Consider the linearized uncoupled stationary problem. Its variational form is: find $\boldsymbol{U} \in W$ such that

$$
A^{\operatorname{lin}}(\boldsymbol{U}, \boldsymbol{V})=L(\boldsymbol{V}) \quad \forall \boldsymbol{V} \in W,
$$

where $W=V_{u} \times V_{p} \times V_{B} \times V_{r}$. If we assume that $\nabla \cdot \boldsymbol{a}=0, \nabla \cdot \boldsymbol{b}=0, A^{\text {lin }}$ satisfies the stability estimate

$$
A^{\operatorname{lin}}(\boldsymbol{U}, \boldsymbol{U})=v\|\nabla \boldsymbol{u}\|^{2}+\frac{1}{\mu_{\mathrm{m}} \rho \mu_{\mathrm{m}} \sigma}\|\nabla \times \boldsymbol{B}\|^{2} .
$$

This stability estimate and the inf-sup conditions between $V_{u}$ and $V_{p}$ and between $V_{B}$ and $V_{r}$, given by

$$
\begin{aligned}
& \inf _{q \in V_{p} \backslash\{0\}} \sup _{\boldsymbol{v} \in V_{u} \backslash\{\boldsymbol{0}\}} \frac{(q, \nabla \cdot \boldsymbol{v})}{\|q\|\|\nabla \boldsymbol{v}\|} \geqslant \beta_{\mathrm{f}}>0, \\
& \inf _{s \in V_{r} \backslash\{0\}} \sup _{\boldsymbol{C} \in V_{B} \backslash\{\boldsymbol{0}\}} \frac{(\nabla s, \boldsymbol{C})}{\|\nabla s\|(\ell\|\nabla \times \boldsymbol{C}\|+\|\boldsymbol{C}\|)} \geqslant \beta_{\mathrm{m}}>0,
\end{aligned}
$$

where $\beta_{\mathrm{f}}$ and $\beta_{\mathrm{m}}$ are constants, are enough to guarantee that the linearized problem is well posed. The parameter $\ell$ has dimensions of length and has been introduced with the only purpose of having a correct scaling for the norm in $V_{B} \subset H($ curl, $\Omega$ ). Therefore, for each iteration $k$, given $\boldsymbol{u}^{k}$ and $\boldsymbol{B}^{k}$ there is a unique $\boldsymbol{U}^{k+1}=\left(\boldsymbol{u}^{k+1}, p^{k+1}, \boldsymbol{B}^{k+1}, r^{k+1}\right)$, solution of the linearized problem (26).

It can be shown that, under the same condition for which the nonlinear problem has a unique solution (see [28]), the sequence $\left\{\boldsymbol{U}^{k}\right\}_{k \geqslant 0}$ converges (strongly) to the (unique) solution of the nonlinear problem. The proof of this result is technical but follows the same strategy as for the stationary Navier-Stokes equations without magnetic coupling.
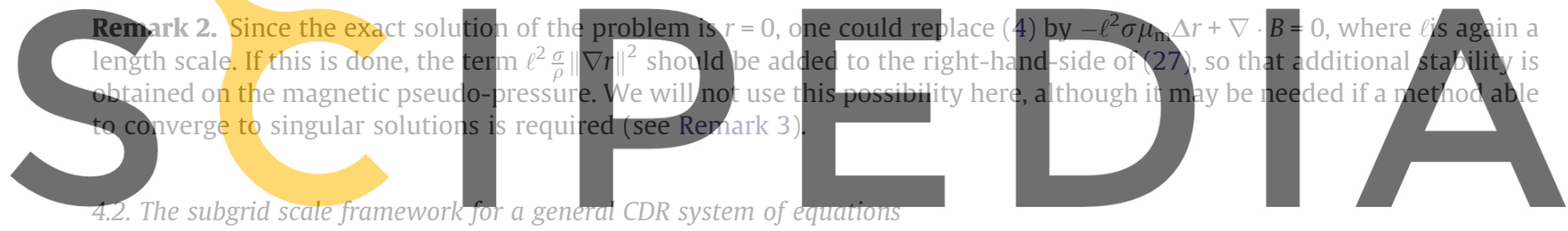

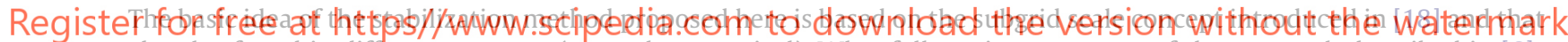
can be also found in different contexts (not only numerical). What follows is a summary of the approach described in [6].

The starting idea is to split the continuous space as $W=W_{h} \oplus \widetilde{W}$, where $W_{h}$ is the finite element space (and therefore finite dimensional) in which the approximate solution will be sought. We call $W$ the space of subscales or subgrid scales. It is readily checked that the continuous problem can be written as the system of equations:

$$
\begin{aligned}
& A^{\operatorname{lin}}\left(\boldsymbol{U}_{h}, \boldsymbol{V}_{h}\right)+A^{\operatorname{lin}}\left(\widetilde{\boldsymbol{U}}, \boldsymbol{V}_{h}\right)=L\left(\boldsymbol{V}_{h}\right) \quad \forall \boldsymbol{V}_{h} \in W_{h}, \\
& A^{\operatorname{lin}}\left(\boldsymbol{U}_{h}, \widetilde{\boldsymbol{V}}\right)+A^{\operatorname{lin}}(\widetilde{\boldsymbol{U}}, \widetilde{\boldsymbol{V}})=L(\widetilde{\boldsymbol{V}}) \quad \forall \widetilde{\boldsymbol{V}} \in \widetilde{W},
\end{aligned}
$$

where $\boldsymbol{U}=\boldsymbol{U}_{h}+\widetilde{\boldsymbol{U}}$ and $\boldsymbol{U}_{h} \in W_{h}, \widetilde{\boldsymbol{U}} \in \widetilde{W}$.

It is useful for the following to introduce the notation

$$
\langle\cdot, \cdot\rangle_{h}:=\sum_{e=1}^{n_{\mathrm{el}}}\langle\cdot, \cdot\rangle_{\Omega^{e}}, \quad\langle\cdot, \cdot\rangle_{\partial h}:=\sum_{e=1}^{n_{\mathrm{el}}}\langle\cdot, \cdot\rangle_{\partial \Omega^{e}},
$$

where $n_{\mathrm{el}}$ is the number of elements of the finite element partition used to build $W_{h}$ and $\Omega^{e}$ denotes the domain of element number $e$.

Integrating by parts all the terms in $A^{\operatorname{lin}}\left(\widetilde{\boldsymbol{U}}, \boldsymbol{V}_{h}\right)$ in (30) and the left-hand-side terms of (31) within each element domain, we get

$$
\begin{aligned}
& A^{\operatorname{lin}}\left(\boldsymbol{U}_{h}, \boldsymbol{V}_{h}\right)+\left\langle\widetilde{\boldsymbol{U}}, n_{i}\left(\boldsymbol{K}_{i j} \partial_{j} \boldsymbol{V}_{h}-\left(\boldsymbol{A}_{i}^{\mathrm{f}}\right)^{\mathrm{t}} \boldsymbol{V}_{h}\right)\right\rangle_{\partial h}+\left\langle\widetilde{\boldsymbol{U}}, \mathcal{L}^{*}\left(\boldsymbol{V}_{h}\right)\right\rangle_{h}=L\left(\boldsymbol{V}_{h}\right), \\
& \left\langle\widetilde{\boldsymbol{V}}, n_{i}\left[\boldsymbol{K}_{i j} \partial_{j}\left(\boldsymbol{U}_{h}+\widetilde{\boldsymbol{U}}\right)-\boldsymbol{A}_{i}^{\mathrm{f}}\left(\boldsymbol{U}_{h}+\widetilde{\boldsymbol{U}}\right)\right]\right\rangle_{\partial h}+\langle\widetilde{\boldsymbol{V}}, \mathcal{L}(\widetilde{\boldsymbol{U}})\rangle_{h}=\left\langle\widetilde{\boldsymbol{V}}, \boldsymbol{F}-\mathcal{L}\left(\boldsymbol{U}_{h}\right)\right\rangle_{h},
\end{aligned}
$$

where $n_{i}$ is now the $i$-th component of the exterior normal to $\partial \Omega^{e}$ and $\mathcal{L}^{*}$ is the adjoint operator of $\mathcal{L}$ with homogeneous Dirichlet conditions, given by 


$$
\mathcal{L}^{*}(\boldsymbol{U}):=-\partial_{i}\left(\boldsymbol{A}_{i}^{\mathrm{t}} \boldsymbol{U}\right)-\partial_{i}\left(\boldsymbol{K}_{i j}^{\mathrm{t}} \partial_{j} \boldsymbol{U}\right) .
$$

Eq. (33) is equivalent to:

$$
\begin{aligned}
& \mathcal{L}(\widetilde{\boldsymbol{U}})=\boldsymbol{F}-\mathcal{L}\left(\boldsymbol{U}_{h}\right)+\boldsymbol{V}_{h, \text { ort }} \text { in } \Omega^{e}, \\
& \widetilde{\boldsymbol{U}}=\widetilde{\boldsymbol{U}}_{\text {ske }} \quad \text { on } \partial \Omega^{e},
\end{aligned}
$$

where $\boldsymbol{V}_{h, \text { ort }}$ is obtained from the condition that $\widetilde{\boldsymbol{U}}$ must belong to $\widetilde{W}$ (and not to the whole space $W$ ) and $\widetilde{\boldsymbol{U}}_{\text {ske }}$ is a function defined on the element boundaries and such that

$$
\mathbf{q}_{n}:=n_{i}\left[\boldsymbol{K}_{i j} \partial_{j}\left(\boldsymbol{U}_{h}+\widetilde{\boldsymbol{U}}\right)-\boldsymbol{A}_{i}^{\mathrm{f}}\left(\boldsymbol{U}_{h}+\widetilde{\boldsymbol{U}}\right)\right]
$$

is continuous across interelement boundaries, and therefore the first term in the left-hand-side of (33) vanishes.

Different subgrid scale (SGS) stabilization methods can be devised depending on the way problem (34), (35) is approximated. The purpose of this paper is not to propose a new methodology, but rather to see how to apply a well established formulation to the incompressible MHD problem. This well known method can be obtained by approximating the subscales by the algebraic expression

$$
\widetilde{\boldsymbol{U}} \approx \tau \widetilde{P}\left[\boldsymbol{F}-\mathcal{L}\left(\boldsymbol{U}_{h}\right)\right]
$$

where $\tau$ is a $n_{\text {unk }} \times n_{\text {unk }}$ matrix of stabilization parameters, the expression of which is discussed in the following subsection, and $\widetilde{P}$ is the projection onto the space of subscales. There are two main possibilities for choosing $\widetilde{P}$ and, therefore, for determining the space of subscales. The most common one is to take $\widetilde{P}=I$, the identity, when applied to the finite element residual appearing in the right-hand-side of (36). Another possibility is to take $\widetilde{P}$ as the $L^{2}$-projection onto the space orthogonal to the finite element space [7,8]. For the sake of conciseness, we will restrict ourselves to the first option $(\widetilde{P}=I)($ see $[11]$ for the analysis of the second approach to time dependent problems). The design of the stabilization parameters, which is our main concern, is the same using both approaches.

To close the approximation, we neglect the interelement boundary terms in (32), which can be understood as taking

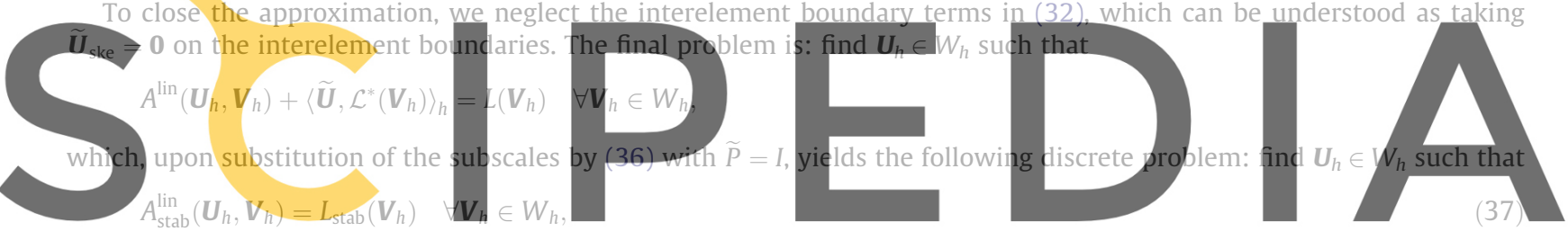

where the bilinear form $A_{\text {stab }}^{\text {lin }}$ and the lineạr form $L_{\text {stab }}$ are now given by

Register for free at https//www.scipedia.com to download the version without the watermark

$$
\begin{aligned}
& A_{\text {stab }}^{\operatorname{lin}}\left(\boldsymbol{U}_{h}, \mathbb{V}_{h}\right)=A^{\operatorname{lin}}\left(\boldsymbol{U}_{h}, \boldsymbol{V}_{h}\right)-\left\langle\mathcal{L}^{*}\left(\mathbb{V}_{h}\right), \tau \mathcal{L}\left(\boldsymbol{U}_{h}\right)\right\rangle_{h}, \\
& L_{\text {stab }}\left(\boldsymbol{V}_{h}\right)=L\left(\boldsymbol{V}_{h}\right)-\left\langle\mathcal{L}^{*}\left(\boldsymbol{V}_{h}\right), \boldsymbol{\tau} \boldsymbol{F}\right\rangle_{h} .
\end{aligned}
$$

\subsection{Stabilized finite element approximation for the linearized MHD problem}

In this subsection we present a stabilized finite element method to approximate problem (26). First of all, we recast it as a system of linear convection-diffusion equations. It is in this general setting that the finite element approximation will be described.

Stabilization for this problem has several goals. The first is to avoid the need to satisfy the discrete counterpart of the infsup conditions (28) and (29), which would lead to different interpolation for the variables of the problem. To fix ideas, we will assume equal and continuous interpolation for all the unknowns. The second goal is to obtain error estimates valid in the limit $v \rightarrow 0$ and $\sigma \mu_{\mathrm{m}} \rightarrow \infty$, that is, for convection-dominated flows (both in the Navier-Stokes equation and the equation for the magnetic field). Finally, the third objective is to account properly for the coupling of the hydrodynamic and the magnetic problems (case $\rho \mu_{\mathrm{m}} \rightarrow 0$ ). That these goals are all satisfied will be seen in the error estimate to be presented.

Up to now we have described the algebraic version of the SGS stabilization in a general setting. The objective now is to apply it to the MHD problem we are considering. In particular, the adjoint operator of the linearized uncoupled MHD problem $\mathcal{L}^{*}\left(\boldsymbol{V}_{h}\right)$, taking once again $\alpha_{B}=1 /\left(\mu_{\mathrm{m}} \rho\right)$, is now given by

$$
\mathcal{L}^{*}(\boldsymbol{V})=\left[\begin{array}{l}
-\boldsymbol{a} \cdot \nabla \boldsymbol{v}-v \Delta \boldsymbol{v}-\nabla q-\frac{1}{\rho \mu_{\mathrm{m}}} \boldsymbol{b} \times(\nabla \times \boldsymbol{C}) \\
-\nabla \cdot \boldsymbol{v} \\
\frac{1}{\rho \mu_{\mathrm{m}}} \nabla \times(\boldsymbol{v} \times \boldsymbol{b})+\frac{1}{\rho \mu_{\mathrm{m}} \sigma \mu_{\mathrm{m}}} \nabla \times(\nabla \times \boldsymbol{C})-\frac{1}{\rho \mu_{\mathrm{m}}} \nabla s \\
-\frac{1}{\rho \mu_{\mathrm{m}}} \nabla \cdot \boldsymbol{C}
\end{array}\right] .
$$


To define the method for the particular MHD problem, an expression for the matrix of stabilization parameters $\tau$ needs to be proposed. To our knowledge, there is no general way to define it for systems of equations [6]. It must be designed for each particular problem taking into account its stability deficiencies.

In the case we are considering, we will see in the following subsection that stability can be improved maintaining optimal accuracy by taking a simple diagonal expression for $\tau$, with one scalar component for each of the equations. In the 3D case, we take

$$
\tau=\operatorname{diag}\left(\tau_{1}, \tau_{1}, \tau_{1}, \tau_{2}, \tau_{3}, \tau_{3}, \tau_{3}, \tau_{4}\right)
$$

Using this expression and (40), it follows that the stabilized bilinear form that we have to consider in problem (37) is

$$
\begin{aligned}
A_{\mathrm{stab}}^{\operatorname{lin}}\left(\boldsymbol{U}_{h}, \boldsymbol{V}_{h}\right)= & A^{\operatorname{lin}}\left(\boldsymbol{U}_{h}, \boldsymbol{V}_{h}\right)-\left\langle\mathcal{L}^{*}\left(\boldsymbol{V}_{h}\right), \tau \mathcal{L}\left(\boldsymbol{U}_{h}\right)\right\rangle=\left\langle\boldsymbol{a} \cdot \nabla \boldsymbol{u}_{h}, \boldsymbol{v}_{h}\right\rangle+v\left(\nabla \boldsymbol{u}_{h}, \nabla \boldsymbol{v}_{h}\right)-\left(p_{h}, \nabla \cdot \boldsymbol{v}_{h}\right)+\left(q_{h}, \nabla \cdot \boldsymbol{u}_{h}\right) \\
& +\frac{1}{\rho \mu_{\mathrm{m}}}\left\langle\boldsymbol{B}_{h}, \nabla \times\left(\boldsymbol{v}_{h} \times \boldsymbol{b}\right)\right\rangle-\frac{1}{\rho \mu_{\mathrm{m}}}\left\langle\boldsymbol{C}_{h}, \nabla \times\left(\boldsymbol{u}_{h} \times \boldsymbol{b}\right)\right\rangle+\frac{1}{\rho \mu_{\mathrm{m}} \sigma \mu_{\mathrm{m}}}\left(\nabla \times \boldsymbol{B}_{h}, \nabla \times \boldsymbol{C}_{h}\right)+\frac{1}{\rho \mu_{\mathrm{m}}}\left(\nabla r_{h}, \boldsymbol{C}_{h}\right) \\
& -\frac{1}{\rho \mu_{\mathrm{m}}}\left(\nabla s_{h}, \boldsymbol{B}_{h}\right)+\left\langle X_{u}\left(\boldsymbol{v}_{h}, q_{h}, \boldsymbol{C}_{h}\right)+v \Delta \boldsymbol{v}_{h}, \tau_{1}\left(X_{u}\left(\boldsymbol{u}_{h}, p_{h}, \boldsymbol{B}_{h}\right)-v \Delta \boldsymbol{u}_{h}\right)\right\rangle_{h}+\left\langle\nabla \cdot \boldsymbol{v}_{h}, \tau_{2}\left(\nabla \cdot \boldsymbol{u}_{h}\right)\right\rangle_{h} \\
& +\left\langle X_{B}\left(s_{h}, \boldsymbol{v}_{h}\right)-\frac{1}{\rho \mu_{\mathrm{m}} \sigma \mu_{\mathrm{m}}} \nabla \times\left(\nabla \times \boldsymbol{C}_{h}\right), \tau_{3}\left(X_{B}\left(r_{h}, \boldsymbol{u}_{h}\right)+\frac{1}{\rho \mu_{\mathrm{m}} \sigma \mu_{\mathrm{m}}} \nabla \times\left(\nabla \times \boldsymbol{B}_{h}\right)\right)\right\rangle_{h} \\
& +\frac{1}{\rho^{2} \mu_{\mathrm{m}}^{2}}\left\langle\nabla \cdot C_{h}, \tau_{4}\left(\nabla \cdot B_{h}\right)\right\rangle_{h},
\end{aligned}
$$

where we have introduced the abbreviations
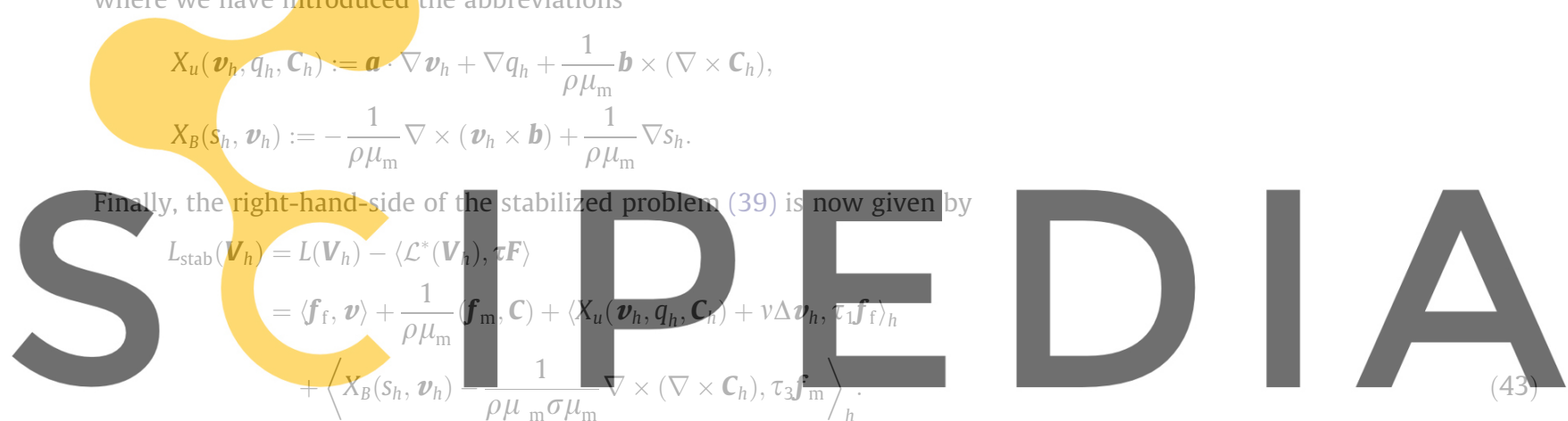

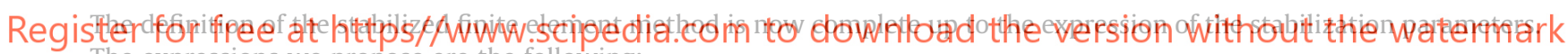
The expressions we propose are the following:

$$
\begin{aligned}
& \alpha:=c_{1} \frac{a}{h}+c_{2} \frac{v}{h^{2}}, \quad \beta:=c_{3} \frac{1}{\rho \mu_{\mathrm{m}}} \frac{b}{h}, \quad \gamma:=c_{4} \frac{1}{\rho \mu_{\mathrm{m}}} \frac{1}{\sigma \mu_{\mathrm{m}}} \frac{1}{h^{2}} \\
& \tau_{1}=\alpha^{-1}\left(1+\frac{1}{\sqrt{\alpha \gamma}} \beta\right)^{-1}, \quad \tau_{2}=c_{5} \frac{h^{2}}{\tau_{1}} \\
& \tau_{3}=\gamma^{-1}\left(1+\frac{1}{\sqrt{\alpha \gamma}} \beta\right)^{-1}, \quad \tau_{4}=c_{6} \rho^{2} \mu_{\mathrm{m}}^{2} \frac{h^{2}}{\tau_{3}}
\end{aligned}
$$

It is understood that these expressions are evaluated element by element. Here, $a$ is the maximum norm of the velocity field $\boldsymbol{a}$ computed in the element under consideration. Likewise, $b$ denotes the maximum norm of $\boldsymbol{b}$ in this element, and $h$ the element diameter. The constants $c_{i}, i=1,2,3,4,5,6$, are independent of the physical parameters of the problem and of the mesh discretization. In the numerical calculations we take them as $c_{1}=2, c_{2}=4, c_{3}=1, c_{4}=4, c_{5}=1, c_{6}=1$.

In the following subsection we justify in detail this choice from the numerical analysis of the problem. We will proceed in a constructive manner, posing conditions on the stabilization parameters obtained from the requirement that the method is stable (coercive) and optimally accurate.

Remark 3. At this point it is important to remark a property of the stabilized formulation we propose concerning convergence to non-regular solutions. In the following analysis we consider the simplest situation, trying to avoid technicalities and focusing our attention on the convenience of the formulation designed. We will state stability of the method and convergence to smooth solutions, assuming all the regularity we need. However, it is known that the problem may exhibit solutions for the magnetic field which are strictly in $H_{0}(\operatorname{curl}, \Omega)$, not even in $\left(H^{1}(\Omega)\right)^{d}$. This happens when $\Omega$ has re-entrant corners [12]. Methods based on penalization, which introduce some sort of control on the divergence of the magnetic field, fail to converge to these non-regular solutions. The remedy is either to weight the terms that give this divergence control, so as to skip it near singularities [13,16], or to use mixed interpolations (including the magnetic 
pseudo-pressure). The former option does not seem easily applicable to general three-dimensional problems, whereas the latter requires the discrete version of the inf-sup condition (29). It is not easy to satisfy it (see [28,17] for possible ways to comply with it). In particular, we are not aware of continuous interpolations satisfying it. Thus, stabilized methods, as the one proposed here, seem to be the only alternative to use the simple continuous Lagrangian approximation of the magnetic field. However, in the formulation we propose the last term in (42) gives also control on the divergence of $B$. In order to converge to non-regular solutions, expressions of $\tau_{4}$ smaller than the one given by (45) might be required.

\subsection{Numerical analysis and design of the stabilization parameters}

In this subsection we proceed to analyze the formulation introduced above and, in particular, to justify the choice (44), (45). For the sake of simplicity in the notation, we will assume that $\boldsymbol{a}$ and $\boldsymbol{b}$ are constant. Likewise, we will assume that the finite element meshes are quasi-uniform. In this case, $h$ in (44), (45) can be taken the same for all the elements (the maximum element diameter), and therefore $\tau_{i}, i=1,2,3,4$, are also constant. Moreover, for quasi-uniform meshes the following inverse estimates hold:

$$
\left\|\nabla v_{h}\right\| \leqslant \frac{C_{\text {inv }}}{h}\left\|v_{h}\right\|, \quad\left\|\nabla \nabla v_{h}\right\| \leqslant \frac{C_{\text {inv }}}{h}\left\|\nabla v_{h}\right\|,
$$

for any function $v_{h}$ in the finite element space and for a certain constant $C_{\text {inv }}$.

The stability and convergence analysis will be made using the following mesh-dependent norm:

$$
\begin{aligned}
\|\| \boldsymbol{U}_{h}\|\|^{2}:= & v\left\|\nabla \boldsymbol{u}_{h}\right\|^{2}+\frac{1}{\rho \mu_{\mathrm{m}} \sigma \mu_{\mathrm{m}}}\left\|\nabla \times \boldsymbol{B}_{h}\right\|^{2}+\tau_{1}\left\|\boldsymbol{a} \cdot \nabla \boldsymbol{u}_{h}+\nabla p_{h}+\frac{1}{\rho \mu_{\mathrm{m}}} \boldsymbol{b} \times\left(\nabla \times \boldsymbol{B}_{h}\right)\right\|^{2}+\tau_{2}\left\|\nabla \cdot \boldsymbol{u}_{h}\right\|^{2} \\
& +\tau_{3} \frac{1}{\rho^{2} \mu_{\mathrm{m}}^{2}}\left\|-\nabla \times\left(\boldsymbol{u}_{h} \times \boldsymbol{b}\right)+\nabla r_{h}\right\|^{2}+\tau_{4} \frac{1}{\rho^{2} \mu_{\mathrm{m}}^{2}}\left\|\nabla \cdot \boldsymbol{B}_{h}\right\|^{2} \\
\equiv & v\left\|\nabla \boldsymbol{u}_{h}\right\|^{2}+\frac{1}{\rho \mu_{\mathrm{m}} \sigma \mu_{\mathrm{m}}}\left\|\nabla \times \boldsymbol{B}_{h}\right\|^{2}+\tau_{1}\left\|X_{u}\left(\boldsymbol{u}_{h}, p_{h}, \boldsymbol{B}_{h}\right)\right\|^{2}+\tau_{2}\left\|\nabla \cdot \boldsymbol{u}_{h}\right\|^{2}+\tau_{3}\left\|X_{B}\left(r_{h}, \boldsymbol{u}_{h}\right)\right\|^{2}+\tau_{4} \frac{1}{\rho^{2} \mu_{\mathrm{m}}^{2}}\left\|\nabla \cdot \boldsymbol{B}_{h}\right\|^{2} .
\end{aligned}
$$

In all what follows, $C$ will denote a positive constant, not necessarily the same at different appearances.

\subsubsection{Coercivity}

Let us start by proving stability in the form of coercivity of the bilinear form (42). It is immediately checked that

$$
\begin{aligned}
A_{\mathrm{stab}}^{\operatorname{lin}}\left(\boldsymbol{U}_{h}, \boldsymbol{U}_{h}\right)= & A^{\operatorname{lin}}\left(\boldsymbol{U}_{h}, \boldsymbol{U}_{h}\right)-\left\langle\mathcal{L}^{*}\left(\boldsymbol{U}_{h}\right), \tau \mathcal{L}\left(\boldsymbol{U}_{h}\right)\right\rangle_{h} \\
= & v\|\nabla \boldsymbol{u}\|^{2}+\frac{1}{\rho \mu_{\mathrm{m}} \sigma \mu_{\mathrm{m}}}\|\nabla \times \boldsymbol{B}\|^{2}+\tau_{1}\left\|X_{u}\left(\boldsymbol{u}_{h}, p_{h}, \boldsymbol{B}_{h}\right)\right\|^{2}-\tau_{1} v^{2}\left\|\Delta \boldsymbol{u}_{h}\right\|^{2}+\tau_{2}\left\|\nabla \cdot \boldsymbol{u}_{h}\right\|^{2}+\tau_{3}\left\|X_{B}\left(r_{h}, \boldsymbol{u}_{h}\right)\right\|^{2} \\
& -\tau_{3} \frac{1}{\rho^{2} \mu_{\mathrm{m}}^{2} \sigma^{2} \mu_{\mathrm{m}}^{2}}\|\nabla \times \nabla \times \boldsymbol{B}\|^{2}+\tau_{4} \frac{1}{\rho^{2} \mu_{\mathrm{m}}^{2}}\left\|\nabla \cdot \boldsymbol{B}_{h}\right\|^{2} .
\end{aligned}
$$

Using the second inverse estimate in (46), it is clear that a sufficient condition for $A_{\text {stab }}^{\text {lin }}$ to be coercive is that

$$
\begin{aligned}
& v-\tau_{1} v^{2} \frac{C_{\mathrm{inv}}^{2}}{h^{2}} \geqslant \alpha v \Longleftrightarrow \tau_{1} \leqslant(1-\alpha) \frac{1}{v} \frac{h^{2}}{C_{\mathrm{inv}}^{2}}, \\
& \frac{1}{\rho \mu_{\mathrm{m}} \sigma \mu_{\mathrm{m}}}-\tau_{3} \frac{1}{\rho^{2} \mu_{\mathrm{m}}^{2} \sigma^{2} \mu_{\mathrm{m}}^{2}} \frac{C_{\mathrm{inv}}^{2}}{h^{2}} \geqslant \alpha \frac{1}{\rho \mu_{\mathrm{m}} \sigma \mu_{\mathrm{m}}} \Longleftrightarrow \tau_{3} \leqslant(1-\alpha) \rho \mu_{\mathrm{m}} \sigma \mu_{\mathrm{m}} \frac{h^{2}}{C_{\mathrm{inv}}^{2}},
\end{aligned}
$$

with $0<\alpha<1$. Conditions (48) and (49) yield

$$
A_{\text {stab }}^{\operatorname{lin}}\left(\boldsymbol{U}_{h}, \boldsymbol{U}_{h}\right) \geqslant\left. C\left|\| \boldsymbol{U}_{h}\right|\right|^{2},
$$

for a constant $C$ independent of the discretization and of the physical parameters (it depends only on the constants of the stabilization parameters).

\subsubsection{Optimal accuracy}

We have obtained conditions (48) and (49) on the stabilization parameters by requiring stability. The rest of conditions will be obtained by imposing that the stabilized method proposed is optimally accurate, which will lead to optimal convergence.

For a function $v$, let $\pi_{h}(v)$ be its optimal finite element approximation. We assume that the following interpolation estimates hold:

$$
\left\|v-\pi_{h}(v)\right\|_{H^{i}(\Omega)} \leqslant \varepsilon_{i}(v):=C h^{k+1-i}|v|_{H^{k+1}(\Omega)}, \quad i=0,1
$$


where $\|\left. v\right|_{H^{q}(\Omega)}$ is the $H^{q}(\Omega)$-norm of $v$, that is, the sum of the $L^{2}(\Omega)$-norm of the derivatives of $v$ up to degree $q$ (and thus the $H^{0}(\Omega)$-norm coincides with the $L^{2}(\Omega)$-norm), $|v|_{H^{q}(\Omega)}$ the corresponding semi-norm, and $k$ is the degree of the finite element approximation.

We will state in the following that the error function of the formulation is

$$
E(h):=\tau_{1}^{-1 / 2} \varepsilon_{0}(\boldsymbol{u})+\tau_{2}^{-1 / 2} \varepsilon_{0}(p)+\tau_{3}^{-1 / 2} \varepsilon_{0}\left(\boldsymbol{B}+\tau_{4}^{-1 / 2} \varepsilon_{0}(r) .\right.
$$

The conditions on the stabilization parameters we will obtain will in fact show that this is indeed the error function and that this error function is optimal.

Let $\boldsymbol{U}$ be the solution of the continuous problem and $\pi_{h}(\boldsymbol{U})$ its optimal finite element approximation. The accuracy estimate that is needed to prove convergence is

$$
A_{\text {stab }}^{\operatorname{lin}}\left(\boldsymbol{U}-\pi_{h}(\boldsymbol{U}), \boldsymbol{V}_{h}\right) \leqslant C E(h)\left\|\left|\boldsymbol{V}_{h}\right|\right\|,
$$

for any finite element function $\boldsymbol{V}_{h}$. The proof of this result can be done using standard arguments in the analysis of finite element formulations and is omitted.

So far, we have not posed any additional conditions on the stabilization parameters other than (48) and (49), found from the requirement of coercivity. The rest of conditions will come from the requirement of optimal accuracy.

It can be shown that

$$
\begin{aligned}
-\left\langle\mathcal{L}^{*}\left(\boldsymbol{V}_{h}\right), \tau \mathcal{L}\left(\boldsymbol{U}-\pi_{h}(\boldsymbol{U})\right)\right\rangle \leqslant & C\left|\left\|\boldsymbol{V}_{h}\left|\left\|\left|\left[\tau_{1}^{1 / 2}\left(\frac{a}{h}+\frac{v}{h^{2}}\right)+\frac{\tau_{3}^{1 / 2}}{\rho \mu_{\mathrm{m}}} \frac{b}{h}+\tau_{2}^{1 / 2} \frac{1}{h}\right] \varepsilon_{0}(\boldsymbol{u})+C\right|\right\| \boldsymbol{V}_{h} \|\right|\left[\tau_{1}^{1 / 2} \frac{1}{h}\right] \varepsilon_{0}(p)\right.\right. \\
& +C||\left|\boldsymbol{V}_{h}\right|\left\|\left[\frac{\tau_{1}^{1 / 2}}{\rho \mu_{\mathrm{m}}} \frac{b}{h}+\frac{\tau_{3}^{1 / 2}}{\rho \mu_{\mathrm{m}}} \frac{1}{\sigma \mu_{\mathrm{m}}} \frac{1}{h^{2}}+\frac{\tau_{4}^{1 / 2}}{\rho \mu_{\mathrm{m}}} \frac{1}{h}\right] \varepsilon_{0}(\boldsymbol{B})+C||\left|\boldsymbol{V}_{h}\right|\right\|\left[\frac{\tau_{3}^{1 / 2}}{\rho \mu_{\mathrm{m}}} \frac{1}{h}\right] \varepsilon_{0}(r)
\end{aligned}
$$

From the definitions (44), (45) of the stabilization parameters it follows that these terms can be bounded also as indicated in (53).

Remark 4. The last step provides in fact the crucial design condition for the stabilization parameters. Expressions (44), (45) follow by imposing that

$$
\begin{aligned}
& \tau_{1}^{1 / 2}\left(\frac{a}{h}+\frac{v}{h^{2}}\right)+\frac{\tau_{3}^{1 / 2}}{\rho \mu_{\mathrm{m}}} \frac{b}{h}+\tau_{2}^{1 / 2} \frac{1}{h} \sim \tau_{1}^{-1 / 2}, \\
& \tau_{1}^{1 / 2} \frac{1}{h} \sim \tau_{2}^{-1 / 2}, \\
& \frac{\tau_{1}^{1 / 2}}{\rho \mu_{\mathrm{m}}} \frac{b}{h}+\frac{\tau_{3}^{1 / 2}}{\rho \mu_{\mathrm{m}}} \frac{1}{\sigma \mu_{\mathrm{m}}} \frac{1}{h^{2}}+\frac{\tau_{4}^{1 / 2}}{\rho \mu_{\mathrm{m}}} \frac{1}{h} \sim \tau_{3}^{-1 / 2}, \\
& \frac{\tau_{3}^{1 / 2}}{\rho \mu_{\mathrm{m}}} \frac{1}{h} \sim \tau_{4}^{-1 / 2},
\end{aligned}
$$

where $\sim$ stands for equality up to constants in each term. The solution of these set of conditions is precisely (44), (45).

\subsubsection{Convergence}

As a consequence of the properties of stability and accuracy in the sense of (53), it is trivial to show that the method is optimally convergent. A trivial check using the expression of the norm ||$|\cdot|||$ given by (47), the interpolation estimates (51) and the stabilization parameters (44), (45) shows that $\left\|\left|\boldsymbol{U}-\pi_{h} \boldsymbol{U} \|\right| \leqslant C E(h)\right.$, from where one can prove using standard arguments that

$$
\left\|\left|\boldsymbol{U}-\boldsymbol{U}_{h} \|\right| \leqslant C E(h) .\right.
$$

The fact that this error estimate is exactly the same as the estimate for the interpolation error $\left\|\left|\boldsymbol{U}-\pi_{h} \boldsymbol{U} \|\right| \leqslant C E(h)\right.$ justifies why it has to be considered 'optimal'. Moreover, a simple inspection of what happens in the limit of dominant second order terms shows that in this case the error estimate reduces to the estimate that could be found using the Galerkin method using finite element spaces satisfying the discrete form of (28), (29), but now, however, using equal interpolation for all the variables. Likewise, in the limit $v \rightarrow 0$ and $\sigma \mu_{\mathrm{m}} \rightarrow \infty$, the error estimate (54) does not blow up and the result can also be considered optimal.

\section{Final numerical scheme}

The final numerical scheme that we propose is obtained by applying the finite element stabilization technique described in Section 4 to the time discrete and linearized problem. The space discretization of these equations, adding stabilization terms as those that appear in (42) and (43) for the stationary thermally uncoupled problem, will lead to the following 
algorithm: For $n=0,1,2, \ldots$, given $\boldsymbol{u}^{n}, p^{n}, \boldsymbol{B}^{n}, r^{n}$ and $\vartheta^{n}$, find $\boldsymbol{u}_{h}^{n+1}, p_{h}^{n+1}, \boldsymbol{B}_{h}^{n+1}, r_{h}^{n+1}$ and $\vartheta_{h}^{n+1}$, as the converged solutions of the following iterative algorithm:

$$
\begin{aligned}
& \left(\delta_{t} \boldsymbol{u}_{h}^{n, k+1}, \boldsymbol{v}_{h}\right)+A_{u u}\left(\boldsymbol{u}_{h}^{n+\theta, k}, \boldsymbol{u}_{h}^{n+\theta, k+1}, \boldsymbol{v}_{h}\right)+A_{u B}\left(\boldsymbol{B}_{h}^{n+\theta, k+1}, \boldsymbol{B}_{h}^{n+\theta, k}, \boldsymbol{v}_{h}\right)+A_{u \vartheta}\left(\vartheta_{h}^{n+\theta, e(k)}, \boldsymbol{v}_{h}\right) \\
& -b_{u}\left(p_{h}^{n+1, k+1}, \boldsymbol{v}_{h}\right)+\left\langle\boldsymbol{u}_{h}^{n+\theta, k} \cdot \nabla \boldsymbol{v}_{h}+v \Delta \boldsymbol{v}_{h}, \tau_{1}^{n+\theta, k} \boldsymbol{R}_{h, u}^{n+\theta, k+1}\right\rangle_{h}+\left\langle\nabla \cdot \boldsymbol{v}_{h}, \tau_{2}^{n+\theta, k} R_{h, p}^{n+\theta, k+1}\right\rangle_{h} \\
& +\left\langle-\alpha_{B} \nabla \times\left(\boldsymbol{v}_{h} \times \boldsymbol{B}^{n+\theta, k}\right), \alpha_{B} \tau_{3}^{n+\theta, k} \boldsymbol{R}_{h, B}^{n+\theta, k+1}\right\rangle_{h}=L_{u}^{n+\theta}\left(\boldsymbol{v}_{h}\right), \\
& b_{u}\left(q_{h}, \boldsymbol{u}_{h}^{n+1, k+1}\right)+\left\langle\nabla q_{h}, \tau_{1}^{n+\theta, k} \boldsymbol{R}_{h, u}^{n+\theta, k+1}\right\rangle_{h}=0, \\
& \alpha_{B}\left(\delta_{t} \boldsymbol{B}^{n, k+1_{h}}, \boldsymbol{C}_{h}\right)+\alpha_{B} A_{B u}\left(\boldsymbol{u}_{h}^{n+\theta, k+1}, \boldsymbol{B}_{h}^{n+\theta, k}, \boldsymbol{C}_{h}\right)+\alpha_{B} A_{B B}\left(\boldsymbol{B}_{h}^{n+\theta, k+1}, \boldsymbol{C}_{h}\right)+\alpha_{B} b_{B}\left(r_{h}^{n+1, k+1}, \boldsymbol{C}_{h}\right) \\
& +\left\langle-\frac{1}{\mu_{\mathrm{m}} \rho}\left(\nabla \times \boldsymbol{C}_{h}\right) \times \boldsymbol{B}_{h}^{n+\theta, k}, \tau_{1}^{n+\theta, k} \boldsymbol{R}_{h, u}^{n+\theta, k+1}\right\rangle_{h}+\alpha_{B}\left\langle\frac{1}{\mu_{\mathrm{m}} \sigma} \nabla \times\left(\nabla \times \boldsymbol{C}_{h}\right), \alpha_{B} \tau_{3}^{n+\theta, k} \boldsymbol{R}_{h, B}^{n+\theta, k+1}\right\rangle_{h} \\
& +\alpha_{B}\left\langle\nabla \cdot \boldsymbol{C}_{h}, \alpha_{B} \tau_{4}^{n+\theta, k} R_{h, r}^{n+\theta, k+1}\right\rangle_{h}=\alpha_{B} L_{B 1}^{n+\theta}\left(\boldsymbol{C}_{h}\right), \\
& \alpha_{B} b_{B}\left(s_{h}, \boldsymbol{B}_{h}^{n+1, k+1}\right)+\alpha_{B}\left\langle\nabla s_{h}, \alpha_{B} \tau_{3}^{n+\theta, k} \boldsymbol{R}_{h, B}^{n+\theta, k+1}\right\rangle_{h}=\alpha_{B} L_{B 2}^{n+\theta}\left(s_{h}\right), \\
& \left(\delta_{t} \vartheta_{h}^{n, k+1}, \psi_{h}\right)+A_{\vartheta u, 1}\left(\boldsymbol{u}^{n+\theta, e^{\prime}(k)_{h}}, \vartheta_{h}^{n+\theta, k+1}, \psi_{h}\right)+A_{\vartheta \vartheta}\left(\vartheta_{h}^{n+\theta, k+1}, \psi_{h}\right)+\left\langle\boldsymbol{u}_{h}^{n+\theta, k} \cdot \nabla \psi_{h}+\frac{k_{\mathrm{t}}}{\rho c_{p}} \Delta \psi_{h}, \tau_{5}^{n+\theta, k} R_{h, \vartheta}^{n+\theta, k+1}\right\rangle_{h} \\
& =L_{T}^{n+\theta, k}\left(\psi_{h}\right),
\end{aligned}
$$

where $\alpha_{B}=1 / \rho \mu_{\mathrm{m}}$ and we have introduced the residuals

$$
\begin{aligned}
\boldsymbol{R}_{h, u} & :=\delta_{t} \boldsymbol{u}_{h}+\boldsymbol{a} \cdot \nabla \boldsymbol{u}_{h}-v \Delta \boldsymbol{u}_{h}+\nabla p_{h}-\frac{1}{\mu_{\mathrm{m}} \rho}\left(\nabla \times \boldsymbol{B}_{h}\right) \times \boldsymbol{b}+\mathbf{g} \beta \vartheta_{h}-\boldsymbol{f}_{\mathrm{f}}, \\
R_{h, p} & :=\nabla \cdot \boldsymbol{u}_{h}, \\
\boldsymbol{R}_{h, B} & :=\delta_{t} \boldsymbol{B}_{h}+\frac{1}{\mu_{\mathrm{m}} \sigma} \nabla \times\left(\nabla \times \boldsymbol{B}_{h}\right)-\nabla \times\left(\boldsymbol{u}_{h} \times \boldsymbol{b}\right)+\nabla r_{h}-\boldsymbol{f}_{\mathrm{m}}, \\
R_{h, r} & :=\nabla \cdot \boldsymbol{B}_{h}, \\
R_{h, \vartheta} & :=\delta_{t} \vartheta_{h}+\boldsymbol{a} \cdot \nabla \vartheta_{h}-\frac{k_{\mathrm{t}}}{\rho c_{p}} \Delta \vartheta_{h}-Q_{\text {tot }} .
\end{aligned}
$$

The superscript $n+\theta, k+1$ in (55)-(59) denotes that these residuals are evaluated with $\boldsymbol{u}_{h}, p_{h}, \boldsymbol{B}_{h}, r_{h}$ and $\vartheta_{h}$ at this time step and iteration counter, whereas now $\boldsymbol{a} \equiv \boldsymbol{u}_{h}^{n+\theta, k}, \boldsymbol{b} \equiv \boldsymbol{B}_{h}^{n+\theta, k}$.

The stabilization parameters $\tau_{i}, i=1,2,3,4$, are given in (44), (45) (and computed within each element), whereas

$$
\tau_{5}=\left(c_{1} \frac{a}{h}+c_{2} \frac{k_{\mathrm{t}}}{\rho c_{p} h^{2}}\right)^{-1} .
$$

Note that the thermal coupling effect has been neglected in the design of the stabilization terms. This approximation is justified in [10].

\section{Numerical examples}

In this section we present five numerical examples designed to show that the formulation presented in this paper satisfies the main requirements posed for its design, namely, it yields smooth solutions for the thermally coupled MHD problem in a wide rank of the physical parameters.

As a general comment from the physical point of view, let us mention that it is well known that the presence of magnetic fields in flows where oscillations can occur precludes their onset. The examples to be presented show clearly this behavior.

\subsection{Convergence test}

In order to analyze the convergence properties of the stabilized finite element approximation presented, a two dimensional problem in the square domain $\Omega=] 0,1[\times] 0,1[$ is considered. This problem was already proposed in [9]. We include it also here to show the behavior of quadratic elements. 
The components of the body forces $\boldsymbol{f}_{\mathrm{f}}=\left(f_{\mathrm{f}, x}, f_{\mathrm{f}, y}\right)$ and $\boldsymbol{f}_{\mathrm{m}}=\left(f_{\mathrm{m}, x}, f_{\mathrm{m}, y}\right)$ are prescribed to:

$$
\begin{aligned}
& f_{\mathrm{f}, x}=f_{1}(x)\left(d_{1}^{\prime}(y)\right)^{2} f_{1}^{\prime}(x)-f_{1}^{\prime}(x) d_{1}(y) f_{1}(x) d_{1}^{\prime \prime}(y)-\frac{1}{\operatorname{Re}}\left[f_{1}^{\prime \prime}(x) d_{1}^{\prime}(y)+f_{1}(x) d_{1}^{\prime \prime \prime}(y)\right]+\mathrm{S}\left[f_{2}^{\prime}(x) d_{2}(y)\left(f_{2}^{\prime \prime}(x) d_{2}(y)+f_{2}(x) d_{2}^{\prime \prime}(y)\right)\right] \\
& f_{\mathrm{f}, y}=-f_{1}(x) d_{1}^{\prime}(y) f_{1}^{\prime \prime}(x) d_{1}(y)+\left(f_{1}^{\prime}(x)\right)^{2} d_{1}(y) d_{1}^{\prime}(y)+\frac{1}{\operatorname{Re}}\left[d_{1}(y) f_{1}^{\prime \prime \prime}(x)+f_{1}^{\prime}(x) d_{1}^{\prime \prime}(y)\right]+\mathrm{S}\left[f_{2}(x) d_{2}^{\prime}(y)\left(f_{2}^{\prime \prime}(x) d_{2}(y)+f_{2}(x) d_{2}^{\prime \prime}(y)\right)\right] \\
& f_{\mathrm{m}, x}=f_{1}(x) f_{2}^{\prime}(x)\left[d_{1}^{\prime \prime}(y) d_{2}(y)+d_{1}^{\prime}(y) d_{2}^{\prime}(y)\right]-f_{1}^{\prime}(x) f_{2}(x)\left[d_{1}^{\prime}(y) d_{2}^{\prime}(y)+d_{1}(y) d_{2}^{\prime \prime}(y)\right]-\frac{1}{\operatorname{Re}_{\mathrm{m}}}\left[f_{2}^{\prime \prime}(x) d_{2}^{\prime}(y)+f_{2}(x) d_{2}^{\prime \prime \prime}(y)\right] \\
& f_{\mathrm{m}, y}=-d_{1}^{\prime}(y) d_{2}(y)\left[f_{1}^{\prime}(x) f_{2}^{\prime}(x)+f_{1}(x) f_{2}^{\prime \prime}(x)\right]+d_{1}(y) d_{2}^{\prime}(y)\left[f_{1}^{\prime \prime}(x) f_{2}(x)+f_{1}^{\prime}(x) f_{2}^{\prime}(x)\right]+\frac{1}{\operatorname{Re}_{\mathrm{m}}}\left[f_{2}^{\prime \prime \prime}(x) d_{2}(y)+f_{2}^{\prime}(x) d_{2}^{\prime \prime}(y)\right]
\end{aligned}
$$

where the prime denotes differentiation. Endowed with this body forces the 2D problem has an exact solution for the velocity given by $\boldsymbol{u}=\left(u_{x}, u_{y}\right)$, where

$$
u_{x}(x, y)=f_{1}(x) d_{1}^{\prime}(y), \quad u_{y}(x, y)=-f_{1}^{\prime}(x) d_{1}(y) .
$$

The analytical solution for the magnetic field is $\boldsymbol{B}=\left(B_{x}, B_{y}\right)$, now with

$$
B_{x}(x, y)=f_{2}(x) d_{2}^{\prime}(y), \quad B_{y}(x, y)=-f_{2}^{\prime}(x) d_{2}(y) .
$$

In this particular example, the functions $f_{1}(x), f_{2}(x), d_{1}(y)$ and $d_{2}(y)$ are chosen as

$$
\begin{array}{ll}
f_{1}(x)=x^{2}(1-x)^{2}, & f_{2}(x)=x^{2}(1-x)^{2}, \\
d_{1}(y)=y^{2}(1-y)^{2}, & d_{2}(y)=y^{2}(1-y)^{2} .
\end{array}
$$

The square domain $\Omega$ has been discretized with five different uniform meshes of $2 \times 25 \times 25,2 \times 50 \times 50,2 \times 75 \times 75$, $2 \times 100 \times 100$ and $2 \times 125 \times 125$ triangular elements, using both linear $\left(P_{1}\right)$ and quadratic $\left(P_{2}\right)$ elements. The characteristic length of the meshes are $h=1 / 25,1 / 50,1 / 75,1 / 100$ and $1 / 125$.

The convergence plots measured in the discrete $L^{2}(\Omega)$-norm for the velocity are shown in Fig. 1 for $\operatorname{Re}=100$ and $\mathrm{Ha}=10$. It is observed that the numerical convergence approximately slope 2 for linear elements and 3 for quadratics, showing that the formulation proposed has optimal convergence behavior.

\subsection{Hartmann problem}

Hartmann flow is a typical benchmark in MHD. It consists in the steady flow of a liquid metal through a channel, in this particular case two-dimensional and without thermal coupling. The liquid flows in the $x$-direction by the influence of a prescribed pressure gradient $G$. A uniform magnetic field $(0,1)$ is applied on the boundaries. The Reynolds number is taken

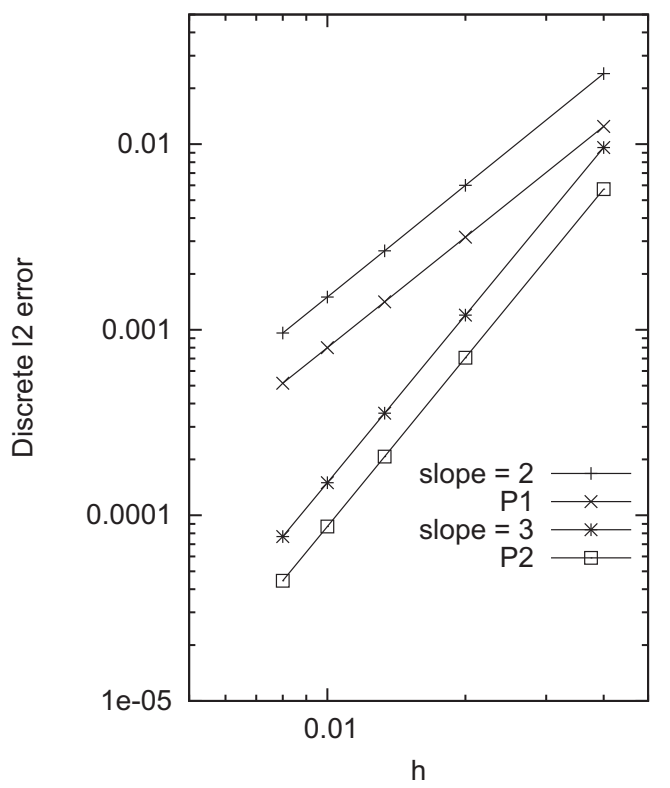

Fig. 1. Velocity error in the discrete $L^{2}$-norm. 
as $\operatorname{Re}=10^{2}$, the magnetic Reynolds number as $\operatorname{Re}_{\mathrm{m}}=10^{-7}$ and different values of $G$ are chosen in such a way that the Hartmann numbers considered are $\mathrm{Ha}=0,10,50$ and 100. The problem definition employed here is the same as in [1].

The flow of the liquid induces a perturbation in the magnetic field in the $x$-direction. The width of the channel is 2 , and we assume that the upper and lower walls are located at $y=1$ and $y=-1$, respectively. In this case, there is an exact solution to the problem, given by

$$
\boldsymbol{u}=\left(u_{x}(y), 0\right), \quad u_{x}(y)=-\frac{G}{\text { Ha } \tanh (\text { Ha })}\left[1-\frac{\cosh (\text { Ha } y)}{\cosh (\text { Ha })}\right] .
$$

This exact velocity profile is prescribed at the inlet and at the outlet of the computational domain. The velocity profile computed at the mid section is shown in Fig. 2, both using bilinear $Q_{1}$ and biquadratic $Q_{2}$ elements on uniform meshes of 40 elements along the $y$-direction. Numerical results for the different Hartmann numbers can be compared with the analytical solution, plotted in solid lines. It is observed that the solution is accurate except close to boundary layers. Since the formulation we have proposed is not intended to be monotone, local oscillations appear close to the walls. It is observed that these are significantly smaller using quadratic elements than linears. The magnetic field (not shown) displays a similar behavior. The local oscillations encountered disappear as the mesh is refined (see [9]).

\subsection{Flow over a circular cylinder}

This numerical simulation was taken from [1]. The problem consists in the flow of a conducting fluid around a 2D circular cylinder while a magnetic field is imposed. From the physical point of view, the main objective in this numerical simulation is to observe the vanishing of the vortexes shed by the cylinder. Our interest is to show that the stabilized formulation we propose yields the correct time response, with the correct coupling between the hydrodynamic and the magnetic problem.

The domain and the finite element mesh used to discretize it are shown in Fig. 3. This mesh is made of 4000 linear triangular elements and 2100 nodes. The boundary conditions for this simulation consist of an imposed constant velocity
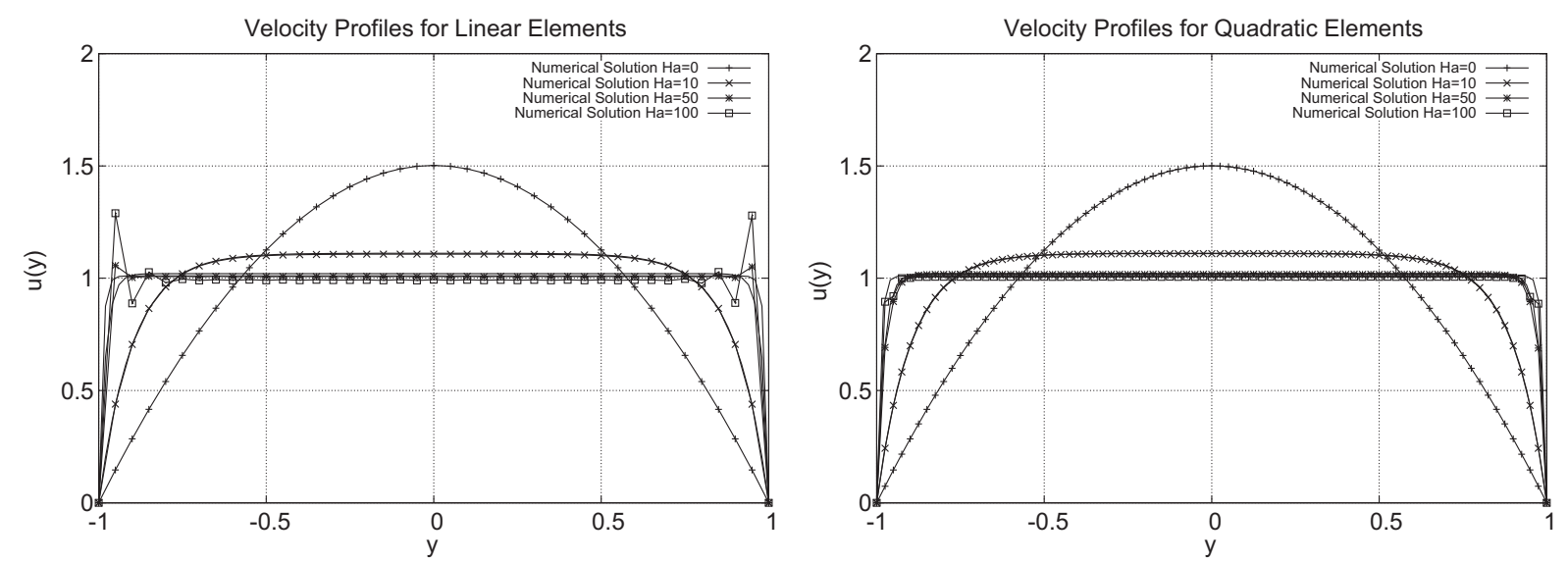

Fig. 2. Hartmann flow. Velocity profiles. Left: linear elements, right: quadratic elements.

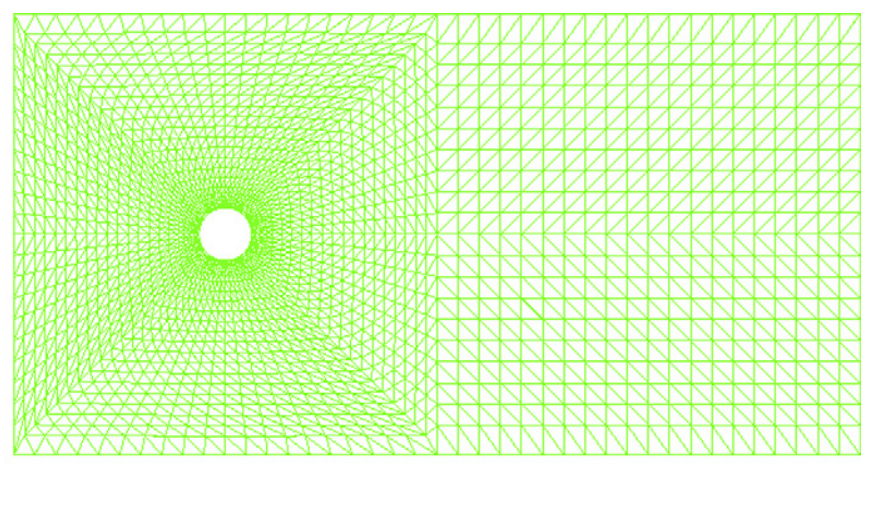

Fig. 3. Flow over a circular cylinder. Domain and finite element mesh. 
at the inlet (left boundary), zero normal velocity on the upper and lower boundaries of the domain, zero velocity on the cylinder and free velocity at the outlet. The boundary conditions for the magnetic field consist of an imposed normal component on the upper and lower boundaries of the domain with zero current (condition (9)), an imposed tangential component at the inlet and the outlet (condition (8)), and a tangent component also on the cylinder.

Numerical simulations have been carried out for $\mathrm{Re}=100$ and the following Hartmann numbers: $\mathrm{Ha}=0.0,1.0,2.5$, and 10.0. These Hartmann numbers are obtained increasing the imposed magnetic field. The magnetic Reynolds Number is $\mathrm{Re}_{\mathrm{m}}=10^{-5}$ and the coupling parameter is taken as $\mathrm{S}=\mathrm{Ha}^{2} / \mathrm{Re}_{\mathrm{m}} \mathrm{Re}$.

The intended effect of the increment in the Hartmann number is to suppress the vortex shedding behind the cylinder. This effect can be observed in Fig. 4, where the contours of the norm of the velocity field at a certain time step are shown for $\mathrm{Ha}=0.0, \mathrm{Ha}=1.0, \mathrm{Ha}=2.5$ and $\mathrm{Ha}=10$. In this last case, not only the shedding has disappeared, but also the vortices have been drastically reduced.
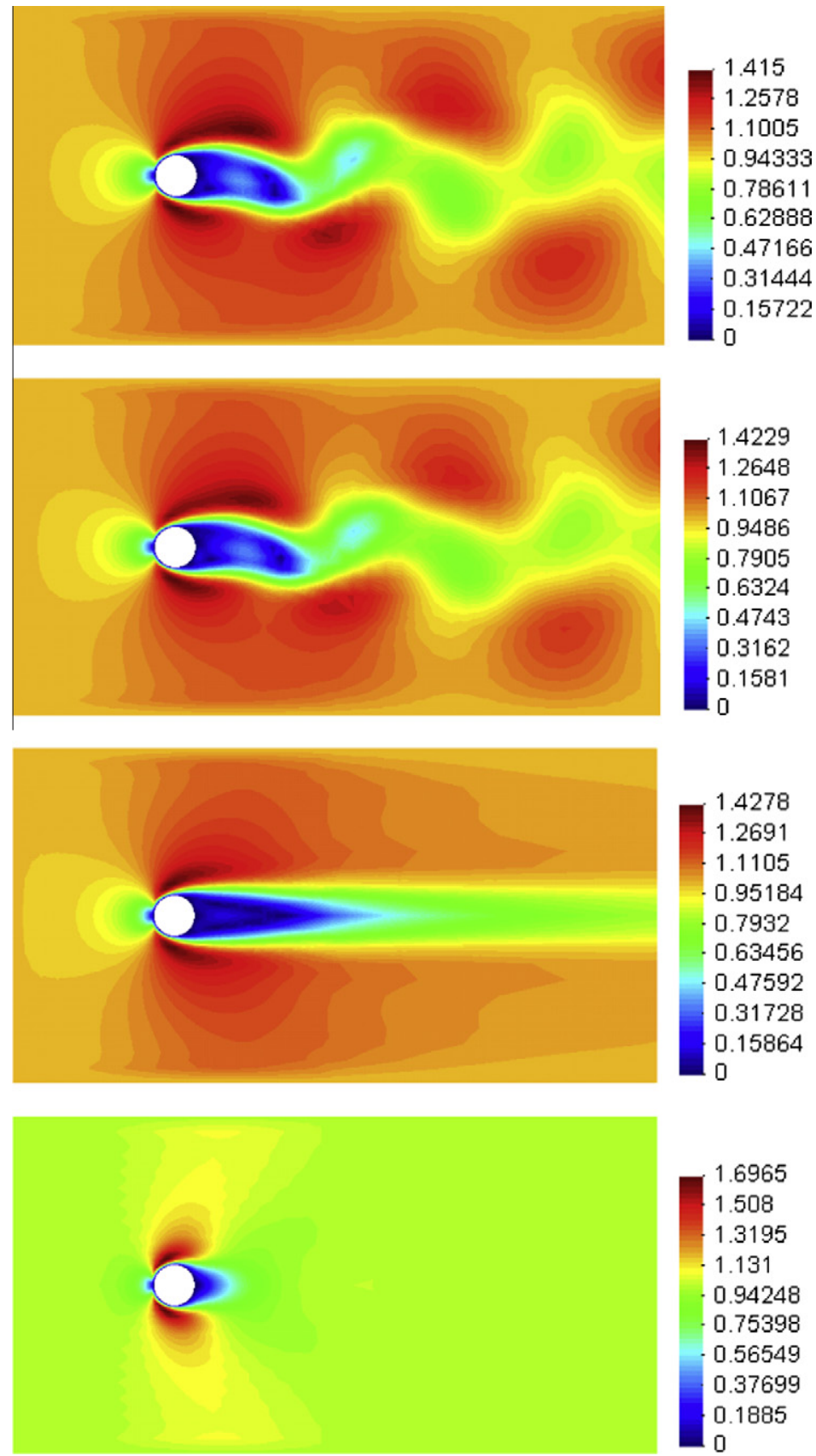

Fig. 4. Flow over a circular cylinder. Velocity contours. From the top to the bottom: $\mathrm{Ha}=0.0,1.0,2.5$ and 10 . 
The suppression of the vortex shedding can also be observed from Fig. 5, where the time evolution of the drag coefficient is plotted for $\mathrm{Ha}=0.0$ and $\mathrm{Ha}=1.0$. The spontaneous shedding occurs approximately at the same time, but the amplitude is clearly higher for $\mathrm{Ha}=0.0$ than for $\mathrm{Ha}=1.0$. At $\mathrm{Ha}=2.5$ it can be seen that the oscillations in the drag coefficient have vanished (not shown).

\subsection{Clogging in continuous casting of steel}

The main objective of this numerical simulation is to observe the behavior of the flow in a continuous casting nozzle while a magnetic field is applied. In operations of continuous casting a very serious problem is the clogging of the nozzles. This is particularly problematic when low carbon steels are casted because some deoxidation products (e.g. alumina) get attached to the walls of the nozzle, forming buildups. These buildups can eventually prevent the flow of steel through the nozzle. This can lead to a decrease in the quality of the steel or even to stop the continuous casting operation and diminish the productivity (see [27]).

The origin of the buildups in the nozzle is associated to the appearance of a recirculation zone at the entry of the nozzle. This recirculation zone is originated by a deattachment of the flow. Although the nozzle can be designed to prevent recirculation, even a small misalignment can originate a deattachment. In order to prevent the recirculation of the flow, the use of a magnetic field has been proposed (see $[20,21]$ ). The magnetic field used to suppress the recirculation is produced by a coil oriented coaxially with the flow. The general effect of the magnetic field is to produce a radial force over the fluid and therefore it tends to attach to the walls of the nozzle.

In order to perform the numerical simulation of the process, a 2D model corresponding to a nozzle section has been constructed. The computational domain considered, as well as the magnetic field prescribed, are shown in Fig. 6 (left). Zero velocity has been prescribed on the walls of the domain. A mesh of 18282 linear triangular elements with 9335 nodes has been used. The following Hartmann numbers have been considered: Ha =1.0, 10.0, 50.0 and 120.0. The Reynolds number that has been taken is $\operatorname{Re} \approx 20000$ and the magnetic Reynolds $\operatorname{Re}_{\mathrm{m}}=0.03632$.

The nature of this example is purely qualitative because there is no numerical benchmark to compare with. The dimensions and general setting of this example have been taken from [21], where the approach to tackle this problem is purely analytical. Due to the dynamical nature of this example the Hartmann number used in order to get a uniform velocity field for the fluid is really high. As it can be seen from Fig. 7, the use of magnetic fields in the nozzle precludes the onset of the recirculation zone. The velocity of the fluid tends to get uniform and therefore the occurrence of buildups is avoided.
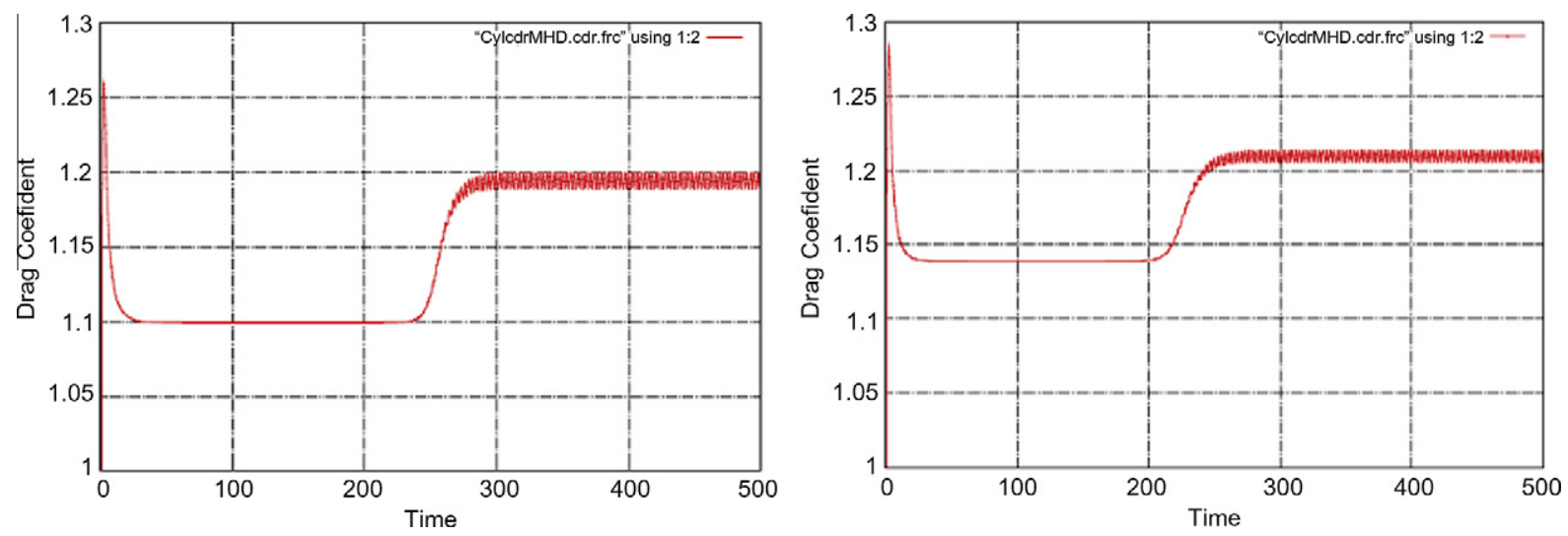

Fig. 5. Flow over a circular cylinder. Time evolution of the drag coefficient for $\mathrm{Ha}=0.0$ (left) and $\mathrm{Ha}=1.0$ (right).
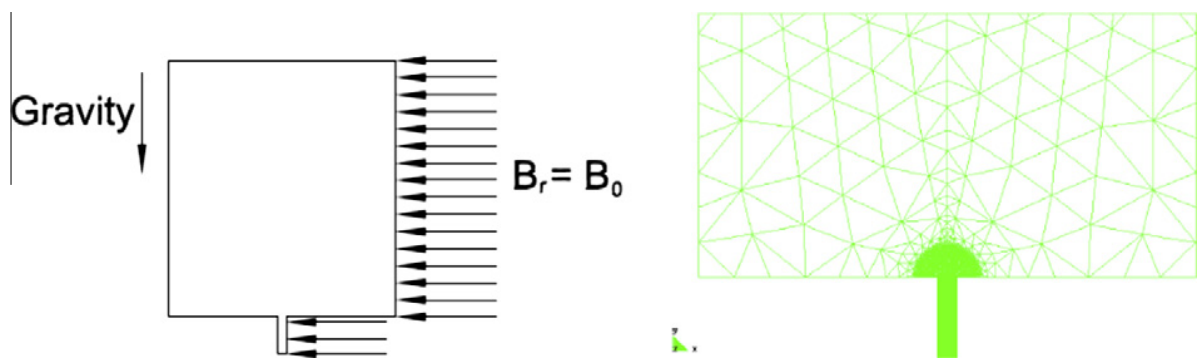

Fig. 6. Clogging in continuous casting. Computational domain (left) and finite element mesh (right). 


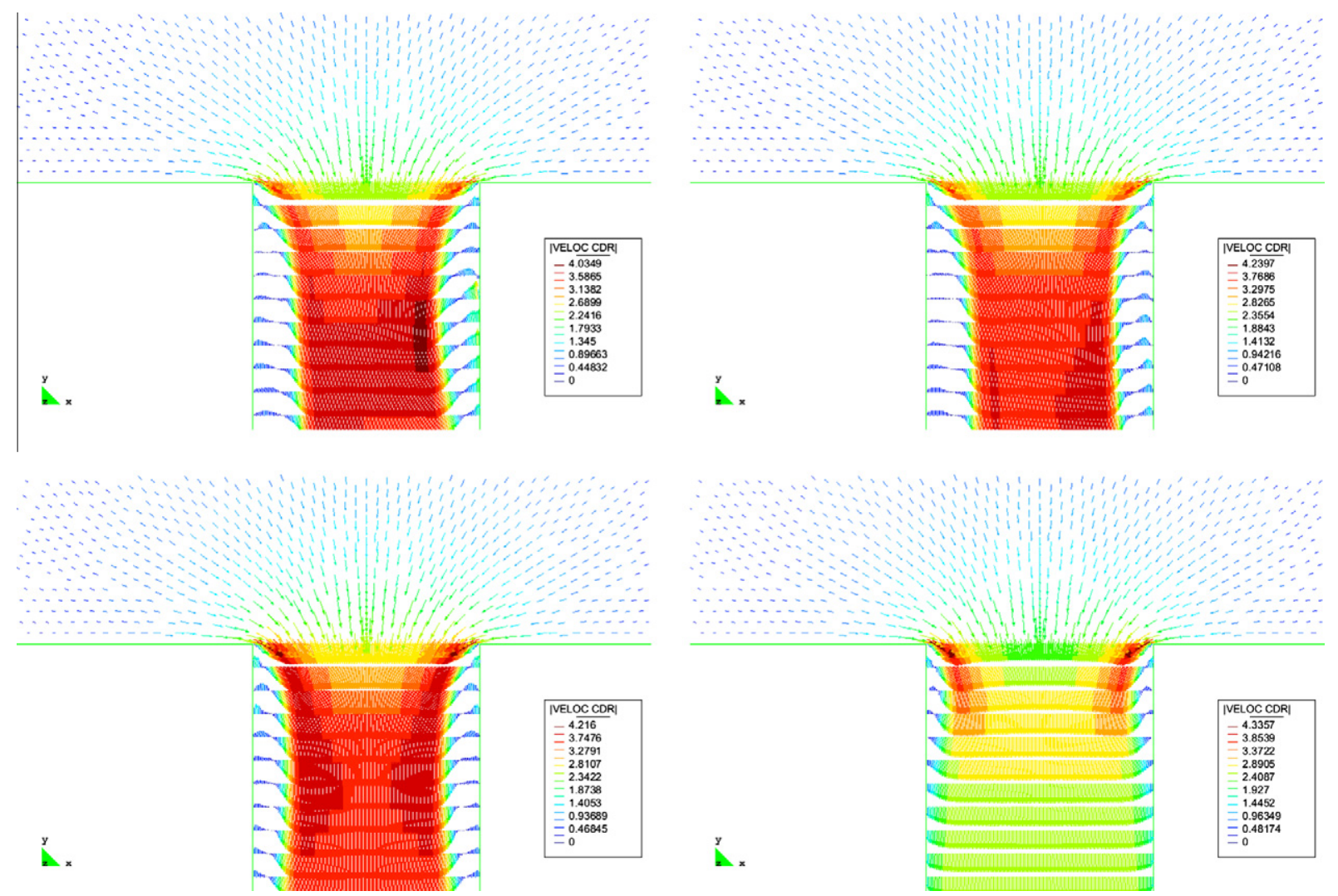

Fig. 7. Clogging in continuous casting. Velocity vectors for different Hartmann numbers. From the left to the right and from the top to the bottom: Ha $=1$, $\mathrm{Ha}=10, \mathrm{Ha}=50$ and $\mathrm{Ha}=120$.

\subsection{Crystal growth}

The main objective of this numerical simulation is to observe the behavior of the molten silicon inside a crucible, in the so called Czochralski process. The numerical simulation to be presented is based on the one proposed by Bückle and Schäfer [3]. The numerical modeling of this crystal growth process is quite complex because it involves a heat transfer problem together with the MHD problem. In the Czochralski process, the heat convection gives rise to fluid motion which can be harmful for the crystalline structure of the silicon. Basically, convection movements can introduce structural defects in the crystal. By applying an intense magnetic field, these convection movements are damped inside the crucible and the defects are diminished or completely eliminated. Cylindrical coordinates were used, assuming the solution to be axisymmetric, although with a non-zero azimuthal velocity. This, together with the axisymmetry of the domain, allowed us to simulate only half of a cross section. A simplified geometry for this problem is presented in Fig. 8.

The general setting for this numerical benchmark is also depicted in Fig. 8. As it can be seen there, the problem consists in a vertical cylindrical crucible filled with molted silicon up to a height $H$, which is rotating with angular velocity $\Omega_{c}$. The coaxial crystal on the top of the crucible is also rotating, but in the sense opposite to the crucible and with angular velocity $\Omega_{X}$. It is assumed that the crystal and the crucible are isothermal, with temperatures $T_{X}$ and $T_{C}$, respectively.

Boundary conditions for this numerical simulation are also indicated in Fig. 8. The boundary conditions for the fluid velocity consist of non-slip conditions on the crucible walls and on the crystal, and a zero traction condition at the surface of the liquid at the top of the crucible. The non-slip boundary condition for the velocity implies that the velocity of the fluid on the crucible walls is the same as the velocity of the walls, and therefore given by the product $\Omega_{C} R_{C}$, where $R_{C}$ is the radius of the crucible. The velocity of the fluid in contact with the crystal is given by $\Omega_{X} R_{X}$, where $R_{X}$ is the radius of the crystal. Concerning the temperature, the boundary conditions are imposed temperature on the crucible walls and on the free surface at the top of the crucible and zero heat flux at the bottom of the crucible. For the magnetic field it is assumed that all interfaces are nonconducting.

In order to completely characterize this numerical simulation, some parameters must be defined. These parameters are the Reynold number for the crucible and the crystal, the Prandtl and Grashof numbers and the aspect ratios, defined as 

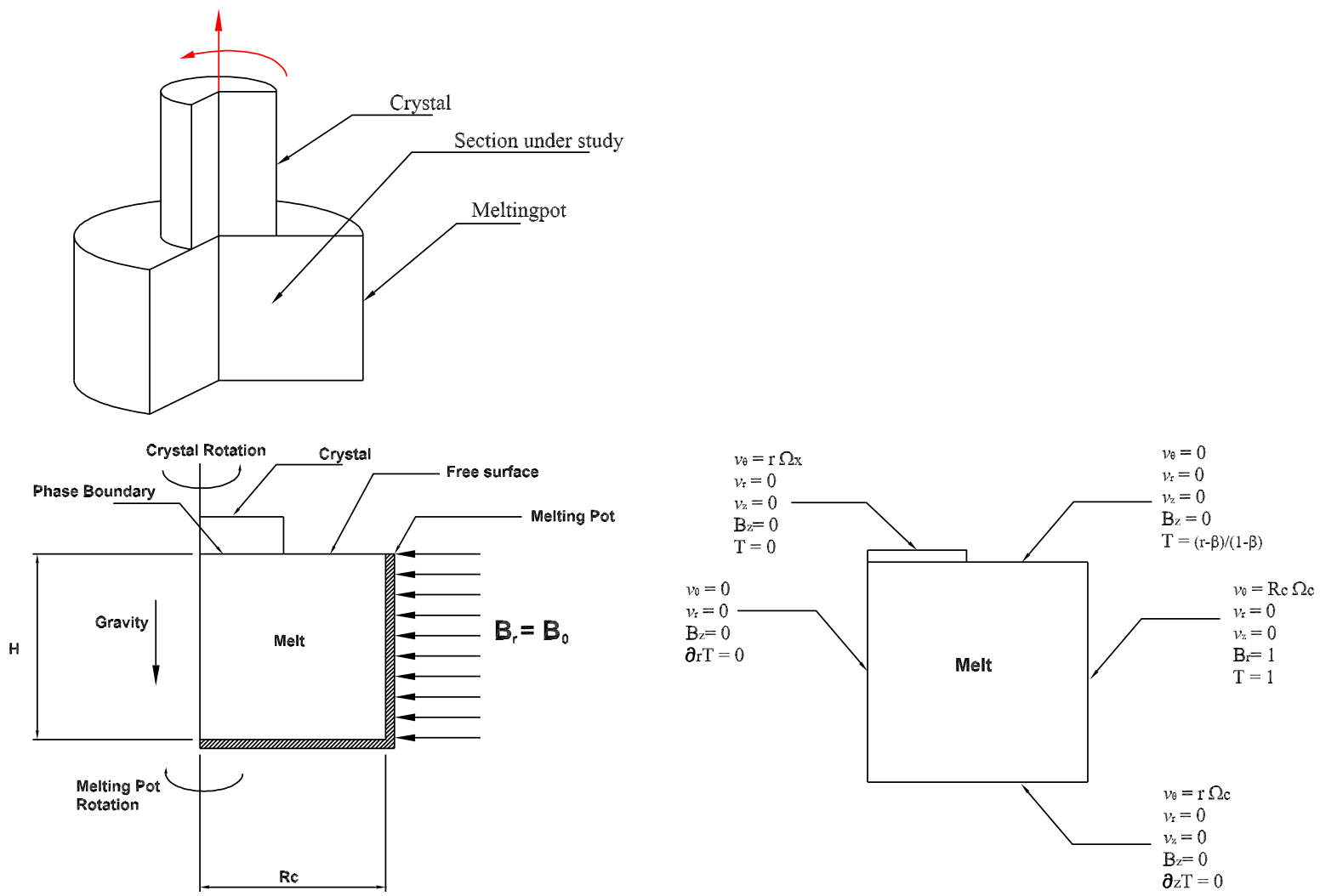

Fig. 8. Crystal growth example. Computational domain (top), cross section (bottom left) and boundary conditions (bottom right).

$$
\begin{aligned}
& \operatorname{Re}_{C}:=\frac{R_{C}^{2} \Omega_{C}}{v}, \quad \operatorname{Re}_{X}:=\frac{R_{X}^{2} \Omega_{X}}{v}, \\
& \operatorname{Pr}:=\frac{v}{\kappa}, \quad \mathrm{Gr}:=\frac{g \beta\left(T_{C}-T_{X}\right) R_{C}^{3}}{v^{2}}, \\
& \hat{\alpha}:=\frac{H}{R_{C}}, \quad \hat{\beta}:=\frac{R_{X}}{R_{C}} .
\end{aligned}
$$

For this particular numerical simulation the aspect ratios and the Prandtl number have been fixed to $\hat{\alpha}=1.0, \hat{\beta}=0.4$ and $\operatorname{Pr}=0.05$. The rest parameters have been defined according to eight cases that were proposed by Bückle and Schäfer in their original paper [3]. Table 1 defines these cases. Although all of them were ran in our numerical testing, in this paper only the results of cases $\mathrm{A} 1$ and $\mathrm{C} 1$ are presented.

The finite element mesh used to discretize the computational domain consists of 5408 linear triangular elements and 2809 nodes, being refined near the boundaries.

Table 1

Values of the physical parameters for the crystal growth example.

\begin{tabular}{llll}
\hline Problem & $\mathrm{Gr}$ & $\mathrm{Re}_{X}$ & $\mathrm{Re}_{C}$ \\
\hline $\mathrm{A} 1$ & 0 & $10^{2}$ & 0 \\
$\mathrm{~A} 2$ & 0 & $10^{3}$ & 0 \\
$\mathrm{~B} 1$ & 0 & $10^{2}$ & $-2.5 \times 10^{1}$ \\
$\mathrm{~B} 2$ & 0 & $10^{3}$ & $-2.5 \times 10^{2}$ \\
$\mathrm{C} 1$ & $10^{3}$ & 0 & 0 \\
$\mathrm{C} 2$ & $10^{4}$ & 0 & 0 \\
D1 & $10^{3}$ & $10^{1}$ & 0 \\
D2 & $10^{4}$ & $10^{2}$ & 0 \\
\hline
\end{tabular}


Fig. 9 (left) shows the velocity vectors for case A1. An increase in the Hartmann number from $\mathrm{Ha}=0.0$ to $\mathrm{Ha}=10.0$ has as a consequence a more uniform velocity field. This uniformity in the velocity field around the crystal prevents the formation of defects in the crystal being created. This also prevents the breaking of the free surface and therefore avoids the inclusion of foreign bodies in the molten silicon.

Fig. 10 shows the distribution of temperature in the molten silicon in the crucible for case $\mathrm{C} 1$. As it can be seen from the pictures, the temperature is mainly convected and the profiles of temperatures are strongly influenced by the velocity of the molten silicon.

Fig. 9 (right) shows the velocity vectors for case C1. As it can be seen in the pictures, the influence of the magnetic field over the velocity is strong enough to diminish the velocity in the crucible. This effect prevents any particle from the walls to get into the area where the crystal is beginning its solidification. We observe therefore that the influence of magnetic fields in the Czochralski process may become an important tool to enhance the quality of crystals made by this process.
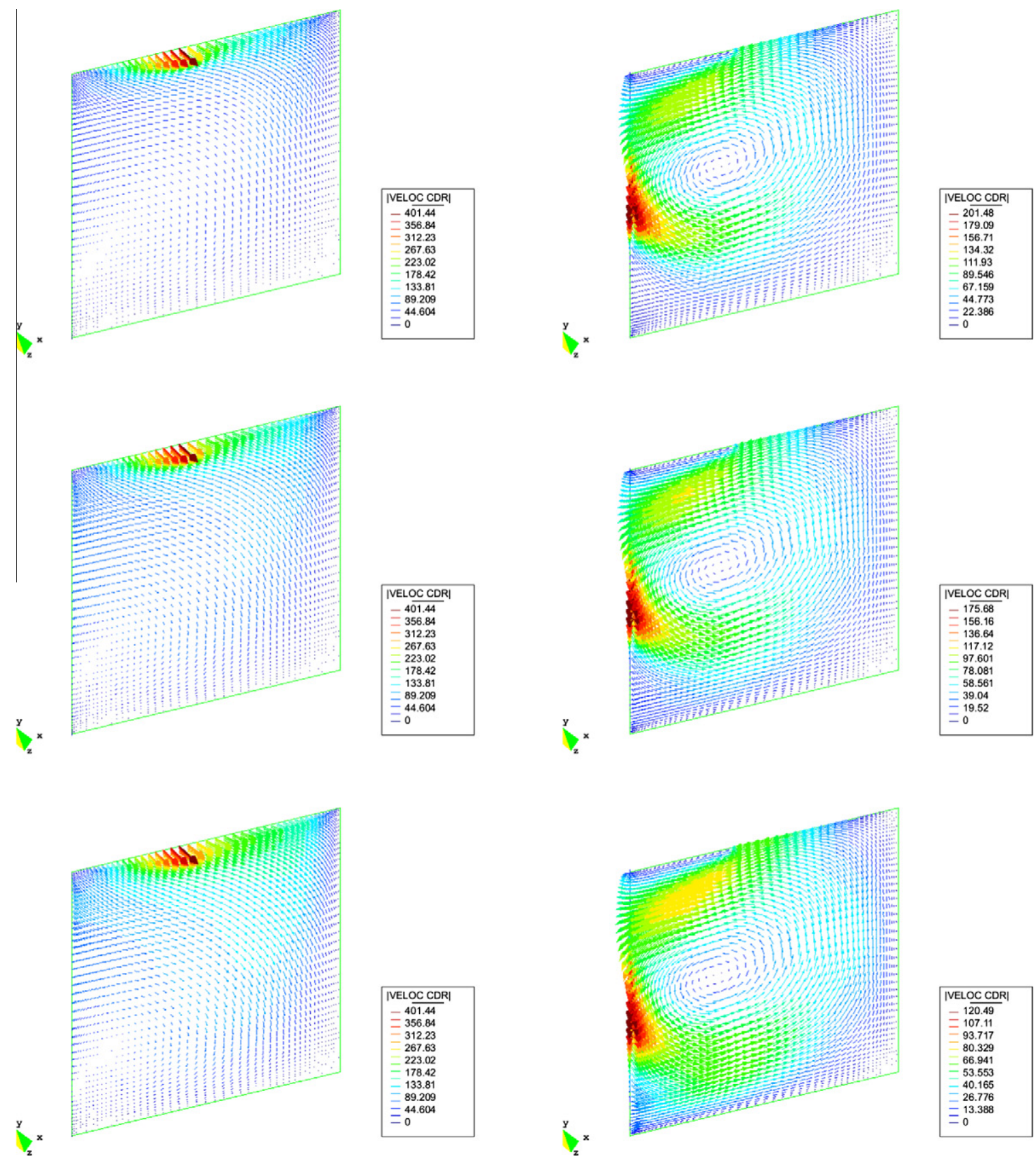

Fig. 9. Crystal growth example. Velocity vectors. Left: case A1, right: case C1. From the top to the bottom: $\mathrm{Ha}=0, \mathrm{Ha}=5 \mathrm{and} \mathrm{Ha}=10$. 


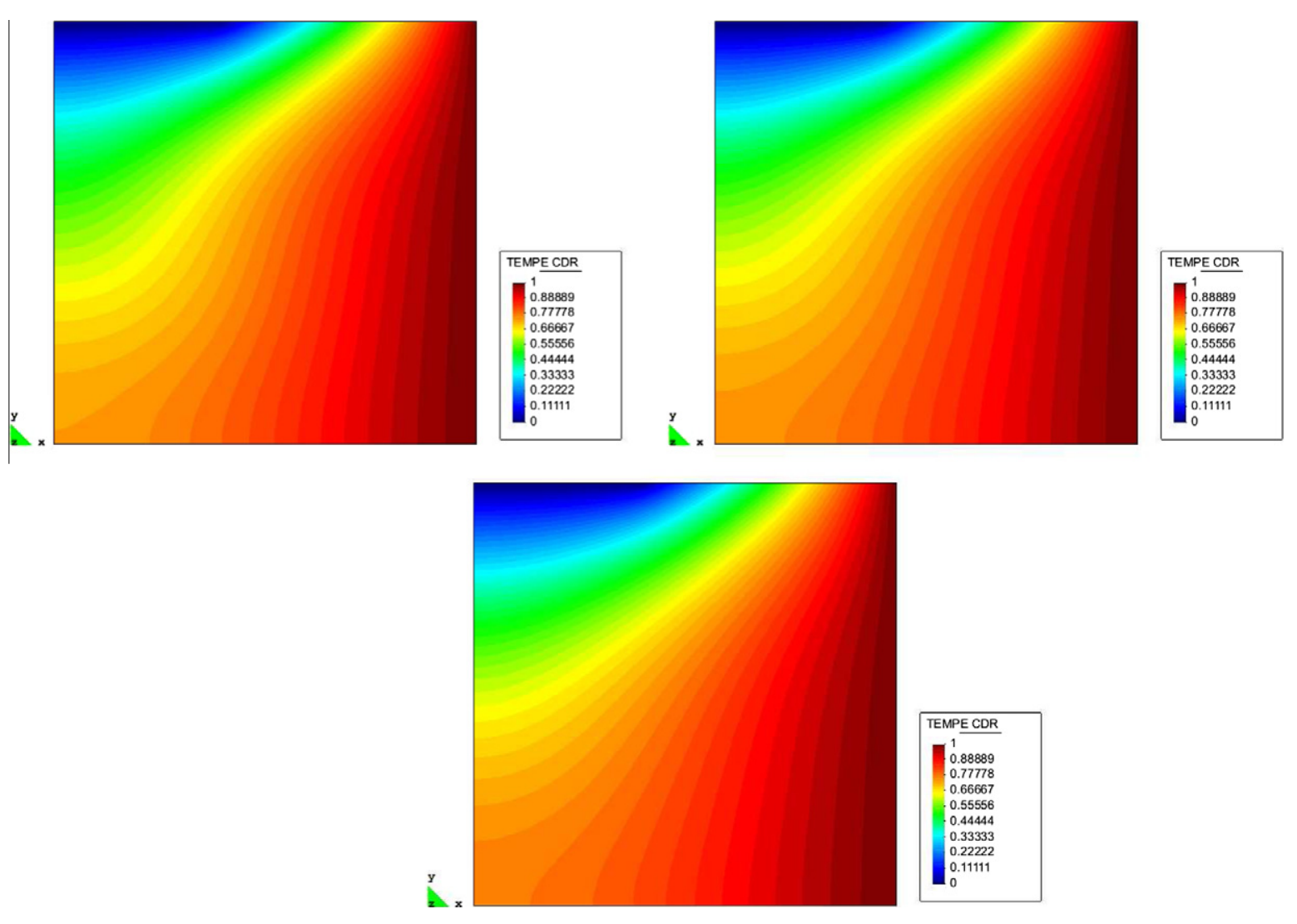

Fig. 10. Crystal growth example. Temperature contours for case $\mathrm{C} 1$. Top left: $\mathrm{Ha}=0$, top right: $\mathrm{Ha}=5$, bottom: $\mathrm{Ha}=10$.

From the numerical point of view, this examples shows the effectiveness of the coupling algorithm proposed to deal with the MHD problem with thermal effects. Referring to this point, Fig. 11 shows the convergence towards the steady state for case D2 with $\mathrm{Ha}=10$, measured as the time evolution of the norm of $\delta_{t} \boldsymbol{U}$ introduced in (18) (with the array of unknowns made dimensionless and the norm normalized with its initial value). This quantity decreases uniformly, until it is below the convergence tolerance chosen of $0.01 \%$. All variables (velocity, pressure, magnetic field, magnetic pseudo-pressure and temperature) are well converged.

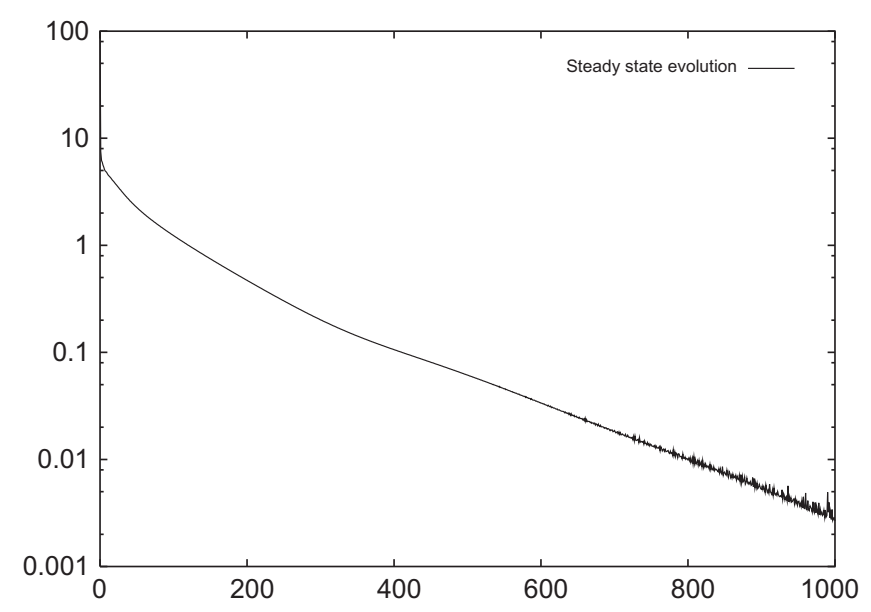

Fig. 11. Crystal growth example. Convergence towards the steady state (case $\mathrm{D} 2, \mathrm{Ha}=10$ ), measured as the norm of $\delta_{t} \boldsymbol{U}$ in time normalized by $\delta_{t} \boldsymbol{U}$ in the first time step and in percentage. 


\section{Conclusions}

In this paper we have presented a numerical formulation to solve thermally coupled MHD flows. It is a stabilized finite element method, whose design is based on splitting the unknown into a finite element component and a subscale and on giving an approximation for the latter. The main features of the formulation are that it allows to use equal interpolation for all the unknowns and it is stable and optimally convergent in a norm that remains meaningful in the whole range of the physical parameters.

Regarding the interpolation issue, it is particularly relevant in problems involving the magnetic field. The method proposed allows us to approximate it using standard continuous interpolations. It has to be remarked that if the solution of the continuous problem exhibits singularities (in non-convex domains), the expression of the stabilization parameters needs to be modified. This is, however, a point that we have not analyzed. Referring to the norm of the stability and error analysis, it gives some sort of control on the unknowns for all values of physical parameters for the linearized problem. Obviously, the fully nonlinear problem may display very complex physics, which need to be approximated not only by a robust formulation, but also by an appropriate discretization. Our objective in this paper has been to address the first point.

The formulation proposed depends on some stabilization parameters. A key point of the work presented here is that these parameters have been designed based on the stability and convergence analysis of the method for the thermally uncoupled problem.

Several computational aspects of the final formulation, such as the time integration, the linearization and the block iterative coupling, have been discussed. Altogether this has led to a method for solving thermally coupled MHD problems that we believe is robust and easy to implement. Some simple numerical examples presented support these statements.

\section{Acknowledgment}

This work has been partially supported by the Consolider-Ingenio project TECNOFUS, Ref. CSD2008-00079, from the Spanish Ministry of Science and Innovation.

\section{References}

[1] F. Armero, J.C. Simo, Long-term dissipativity of time-stepping algorithms for an abstract evolution equation with application to MHD and NavierStokes equations, Computer Methods in Applied Mechanics and Engineering 131 (1996) 41-90.

[2] F. Brezzi, M. Fortin, Mixed and Hybrid Finite Element Methods, SpringerVerlag, 1991.

[3] U. Bückle, M. Schäfer, Benchmark results for the numerical simulation of flow in czochralski crystal growth, Journal of Crystal Growth 126 (1993) $682-$ 694.

[4] M. Cervera, R. Codina, M. Galindo, On the computational efficiency and implementation of block-iterative algorithms for nonlinear coupled problems, Engineering Computations 13 (6) (1996) 4-30.

[5] P. Ciarlet Jr, Augmented formulations for solving Maxwell equations, Computer Methods in Applied Mechanics and Engineering 194 (2005) 559-586.

[6] R. Codina, On stabilized finite element methods for linear systems of convection-diffusion-reaction equations, Computer Methods in Applied Mechanics and Engineering 188 (2000) 61-82.

[7] R. Codina, Stabilized finite element approximation of transient incompressible flows using orthogonal subscales, Computer Methods in Applied Mechanics and Engineering 191 (2002) 4295-4321.

[8] R. Codina, Analysis of a stabilized finite element approximation of the Oseen equations using orthogonal subscales, Applied Numerical Mathematics 58 (2008) 264-283.

[9] R. Codina, N. Hernández, Stabilized finite element approximation of the stationary MHD equations, Computational Mechanics 38 (2006) $344-355$.

[10] R. Codina, J. Principe, M. Avila, Finite element approximation of turbulent thermally coupled incompressible flows with numerical sub-grid scale modeling, International Journal for Numerical Methods for Heat \& Fluid Flow 20 (2010) 492-516.

[11] R. Codina, J. Principe, O. Guasch, S. Badia, Time dependent subscales in the stabilized finite element approximation of incompressible flow problems, Computer Methods in Applied Mechanics and Engineering 196 (2007) 2413-2430.

[12] M. Costabel, M. Dauge, Singularities of electromagnetic fields in polyhedral domains, Archives for Rational Mechanics and Analysis 151 (2000) $221-$ 276.

[13] M. Costabel, M. Dauge, Weighted regularization of Maxwell equations in polyhedral domains, Numerische Mathematik 93 (2002) $239-277$.

[14] L. Demkowicz, L. Vardapetyan, Modeling of electromagnetic absorption/scattering problems using hp-adaptive finite elements, Computer Methods in Applied Mechanics and Engineering 152 (1998) 103-124.

[15] J.F. Gerbeau, A stabilized finite element method for the incompressible magnetohydrodynamic equations, Numerische Mathematik 87 (2000) $83-111$.

[16] U. Hasler, A. Schneebeli, D. Schötzau, Mixed finite element approximation of incompressible MHD problems based on weighted regularization, Applied Numerical Mathematics 51 (2004) 19-45.

[17] P. Houston, I. Perugia, D. Schötzau, Mixed discontinuous Galerkin approximation of the Maxwell operator, SIAM Journal on Numerical Analysis 42 (2004) 434-459.

[18] T.J.R. Hughes, Multiscale phenomena: Green's function the Dirichlet-to-Neumann formulation, subgrid scale models, bubbles and the origins of stabilized formulations, Computer Methods in Applied Mechanics and Engineering 127 (1995) 387-401.

[19] B. Jiang, J. Wu, L.A. Povinelli, The origin of spurious solutions in computational electromagnetics, Journal of Computational Physics 125 (1996) $104-$ 123.

[20] L.I. Kadar, P.P. Biringex, J.D. Lavers, Modification of the nozzle flow using electromagnetic induction, IEEE Transactions on Magnetics 30 (1994) $4686-$ 4688.

[21] J.D. Lavers, L.I. Kadar, Application of electromagnetic forces to reduce tundish nozzle clogging, Applied Mathematical Modelling 28 (2004) 29-45

[22] M.D. Gunzburger, A.J. Meir, J.P. Peterson, On the existence, uniqueness, and finite element approximation of solutions of the equations of stationary, incompressible magnetohydrodynamics, Mathematics of Computation 56 (1991) 523-563.

[23] A. Soulaimani, N.B. Salah, W.G. Habashi, A finite element method for magnetohydrodynamics, Computer Methods in Applied Mechanics and Engineering 190 (2001) 5867-5892.

[24] W.G. Habashi, N.B. Salah, A. Soulaimani, M. Fortin, A conservative stabilized finite element method for the magneto-hydrodynamic equations, International Journal for Numerical Methods in Fluids 29 (1999) 535-554.

[25] J.C. Nédelec, Mixed finite elements in $\mathbb{R}^{3}$, Numerische Mathematik 35 (1980) 315-341. 
[26] J.C. Nédelec, Éléments finis mixtes incompressibles pour l'equation de Stokes dans $\mathbb{R}^{3}$, Numerische Mathematik 39 (1982) 97-112.

[27] K.G. Rackers, B.G. Thomas, Clogging in continuous casting nozzles, in: 78th Steelmaking Conference Proceedings, Iron and Steel Society, Nashville TN., 1995, pp. 723-734.

[28] D. Schötzau, Mixed finite element methods for stationary incompressible magneto-hydrodynamics, Numerische Mathematik 96 (2004) $771-800$.

[29] L. Vardapetyan, L. Demkowicz, hp-adaptive finite elements in electromagnetics, Computer Methods in Applied Mechanics and Engineering 169 (1999) 331-344. 